\title{
Penetration of supersonic gas jets into a tokamak
}

\author{
V. Rozhansky ${ }^{*}$, I. Senichenkov ${ }^{*}$, I. Veselova*, D. Morozov*, R. Schneider ${ }^{* * *}$ \\ *St.Petersburg State Polytechnical University, 195251 St.Petersburg, Russia \\ ** Institute of Nuclear Fusion, RRC 'Kurchatov Institute', Moscow, Russia \\ ${ }^{* * *}$ Max-Planck Institut für Plasmaphysik, Teilinstitut Greifswald, Euroatom Association, \\ D-17491 Greifswald, Germany
}

The injection of high-pressure supersonic jets into the tokamak plasma is considered as a promising_way of future thermonuclear reactor fueling and as a tool for disruption mitigation. Successful experiments were performed on Tore Supra and DIII-D, correspondingly. In the present paper the evolution of such a jet is analyzed. The jet expansion, deceleration of the ambient electrons and ions by the jet, self-consistent electric field, elementary processes, radiation and adiabatic cooling of the ambient plasma are taken into account. The jet is simulated by a MHD code, which is similar to the code previously used for pellets. It is demonstrated that the ionization degree of the jet strongly depends on the jet parameters. Several simulations were performed for the range of parameters typical for DIII-D. The jet of initial density $4 \cdot 10^{24} \mathrm{~m}^{-3}$ remains almost neutral, and only the outer regions are ionized. When the initial jet density is reduced by a factor of 2 or more the main part of the jet becomes ionized rather fast. It is demonstrated that ionization at the jet edge in poloidal (perpendicular to the magnetic field) direction of the jet is sufficient to stop poloidal expansion of the jet by $\vec{j} \times \vec{B}$ force. The final poloidal size of the jet remains of the order of its initial poloidal dimension (of the order of ten centimetres). The jet motion in the radial direction (direction of the injection) is provided by the polarization poloidal electric field and the correspoding $\vec{E} \times \vec{B}$ drift. In the paper two mechanisms of polarization reduction are considered: Alfvén conductivity of the ambient plasma and the $\nabla B$-induced drift. It is shown that almost neutral jet can penetrate deep into the tokamak while a modest ionization degree should prevent its penetration for the case of low field side (LFS) injection. 


\section{Introduction}

Injection of supersonic gas jet is considered to be a new technique of material delivery into a tokamak. This technique was suggested for the future reactor and for disruption mitigation. The general idea is to inject a rather dense jet, which contains large amount of particles, so that it could remain almost neutral in spite of the energetic ambient plasma particles and therefore should be able to penetrate deep into a tokamak. Another advantage of this technique is that it is almost as cheap as gas puff (much cheaper than the pellet injection), but using a Laval nozzle it is possible to achieve a well-collimated jet moving with rather high (supersonic) velocity. In the Figure 1 the jet injected into a tokamak plasma is schematically shown.

The first experiments on jet fuelling were performed on Tore Supra [1,2] and on tokamaks HT-7 [3] and HL-1M [4]. The fuelling efficiency value was reported to be intermediate between the standard gas puff and pellet injection from the high field side (HFS) of the tokamak. The first experiments on disruption mitigation were performed on DIII-D [5,6]. Several gases were used: deuterium $\left(\mathrm{D}_{2}\right)$, helium $(\mathrm{He})$, neon $(\mathrm{Ne})$ and argon (Ar). It was reported that the impurities in high-density jet $\left(n=4 \cdot 10^{24} \mathrm{~m}^{-3}\right)$ penetrate deep into the tokamak plasma (up to $r / a=(0.3 \div 0.5)$, where $r$ is the distance from the magnetic axis, and $a$ is the distance up to the separatrix), causing a thermal and current quench without significant damaging effects, such as thermal loads to the first wall and divertor plates, vessel strength from poloidal halo currents and runaway generation.

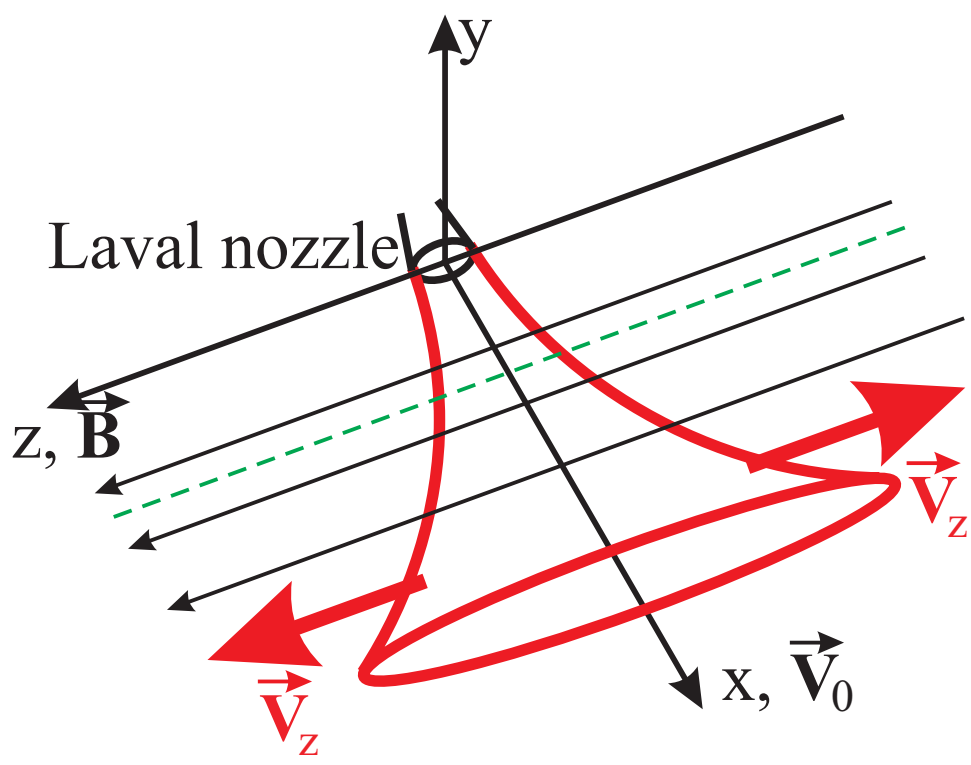

Figure 1. Injection geometry. Shown are the Laval nozzle outside the plasma, the coordinate system used below in the text, and the jet expanding mainly along the magnetic field lines with velocity $\vec{V}_{z}$ (see explanation in the text below). The jet initial velocity $\vec{V}_{0}$ is directed along the $x$ axis. The green dashed line represents the last closed magnetic surface.

In the second series of experiments [7] on DIII-D with lower jet density no significant penetration much past the edge pedestal has been observed but the effect on the discharge was similar. Therefore the highpressure noble gas injection is considered to be a promising technique for disruption mitigation for ITER.

On the ASDEX-Upgrade the supersonic gas jet with the same parameters as on Tore Supra [1,2] was used to control the edge density profiles and to cause ELM event and to control its intensity $[8,9,10]$. No density rise inside the separatrix was observed, which indicates that such a jet does not penetrate into the tokamak.

Up to now the theoretical description of gas jet behavior was absent. The natural interpretation of the deep jet penetration is that the jet remains almost neutral and hence is able to move across magnetic field with the initial velocity. On the other hand the strong energy fluxes coming from the ambient plasma should provide heating, which might be sufficient for at least partial ionization of the jet. Whether the jet remains neutral or ionized depends on the balance of heating power and energy losses spent on the jet expansion, ionization and radiation. 
In the present paper the jet evolution is analyzed. The studying of heating and expansion is performed for a single plasma blob with density, temperature and size equal to those of the jet, while considering the jet penetration and background plasma cooling, the jet is treated as a succession of such blobs. The 1D jet expansion along the magnetic field lines is simulated by means of MHD code, which is similar to the pellet code [11]. MHD parallel expansion, deceleration of the ambient electrons and ions by the jet, self-consistent electric field, elementary processes and radiation are taken into account. Simulations were performed for different gases $\left(D_{2}, A r\right)$, different values of jet density and background plasma density and temperature. It is demonstrated that the ionization degree of the jet strongly depends on the parameters, especially on the jet initial density. For the Argon jet and the parameters of experiment [3]-[4] the main part of the jet remains neutral and only the front expanding along magnetic field is ionized. When the initial jet density is reduced by a factor of 2 or more the main part of the jet becomes ionized rather fast. The jet of lower density, injected to the Tore Supra [1,2] or ASDEX-Upgrade [8-10], is ionized in the separatrix vicinity.

The perpendicular (poloidal) evolution of the jet is also simulated. It is demonstrated that ionization at the edge of the jet is sufficient to stop poloidal expansion of the jet by $\vec{j} \times \vec{B}$ force, Fig 2 . The final poloidal size of the jet remains of the order of its initial poloidal dimension (of the order of ten centimeters). The jet motion in the radial direction (direction of the injection) is more complicated. It is shown that in spite of the fact that the jet pressure gradient is balanced by $\vec{j} \times \vec{B}$ force as in the poloidal direction, the radial penetration may be still possible due to the polarization electric field and corresponding $\vec{E} \times \vec{B}$ drift in the radial direction. It is shown schematically in Fig 3, here $E_{0}=V_{0} B$, where $V_{0}$ is the injection velocity. In the paper two mechanisms of polarization reduction are considered: Alfvén conductivity [12] of the ambient plasma and $\nabla B$-induced drift. Both mechanisms are similar to those discussed in $[13,14]$ for pellet clouds and are responsible for the jet deceleration or acceleration towards the low field side direction.

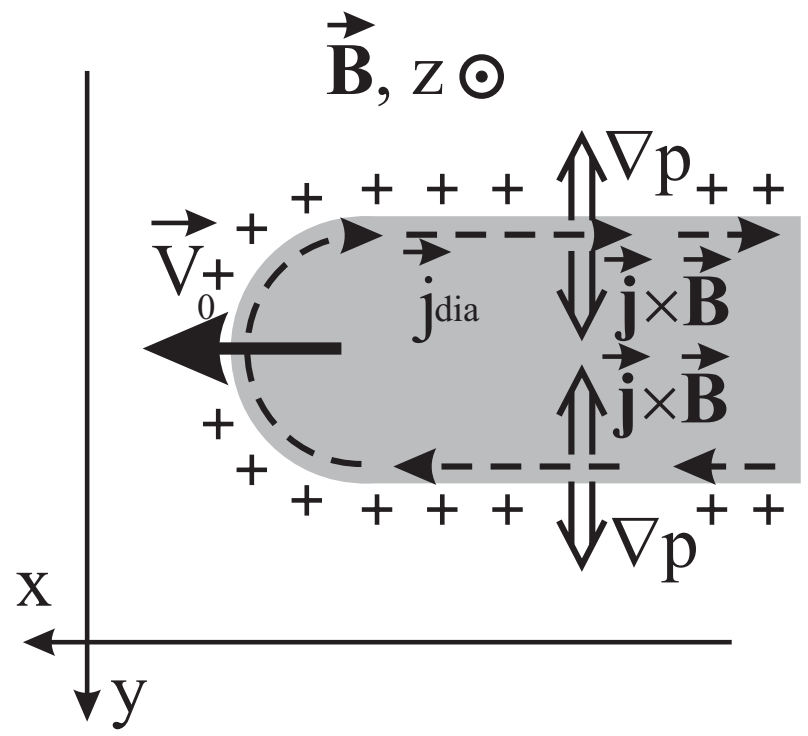

Figure 2. Momentum balance in the poloidal direction. The '+' signs shows a direction of the electric field providing a confinement across the magnetic field, see Sec. 5. Note that the diamagnetic current closes its loop (which is not fully shown in the Figure) flowing along surfaces $p_{e}+p_{i}=$ const .

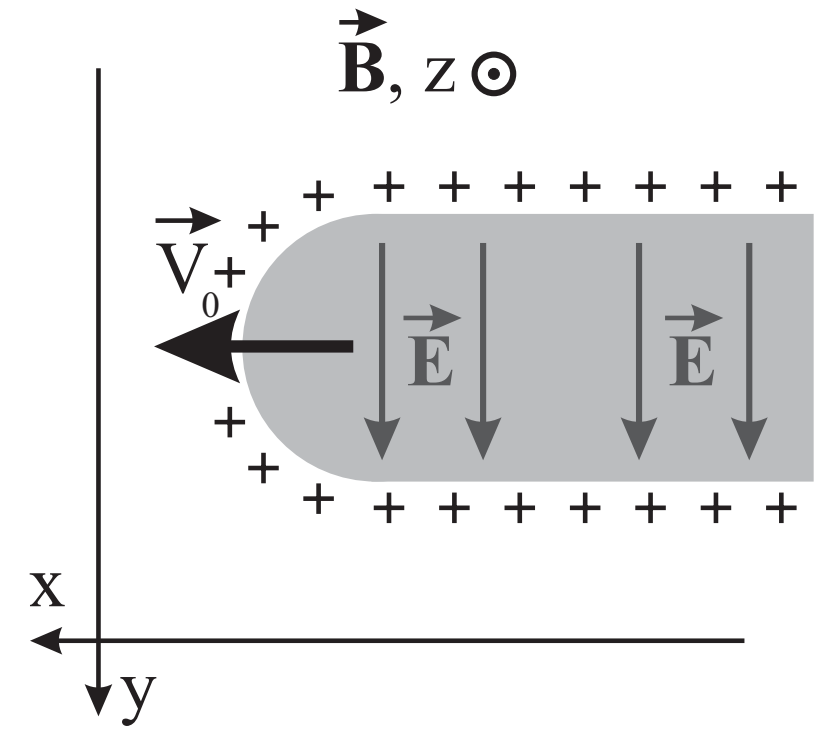

Figure 3. Electric field of dipole type providing the motion across the magnetic field lines. The '+' signs shows a direction of the electric field providing a confinement across the magnetic field, see Sec. 5. 
Recently a theoretical study of fuelling jet penetration was performed by Parks et al. [15]. They studied the fueling jet injected from the high field side of a tokamak. We confirm their result that only a HFS fuelling jet may be able to penetrate deep into the plasma due to $\vec{E} \times \vec{B}$ drift.

The paper is organized as follows. In the Section 2 the gas flow from the Laval nozzle is considered. In the Section 3 the heating and expansion of a single partially ionized plasma blob along the magnetic field lines are discussed. Later the jet is considered as a sequence of such blobs. In the Section 4 results of numerical modeling of the expansion along the magnetic field are presented. In the Section 5 the transverse expansion of partially ionized blob and its confinement in the poloidal direction is studied. In the Section 6 the model for the transverse expansion and confinement in the poloidal direction is described and results of calculations are presented. The Section 7 is devoted to the analysis of the poloidal electric field formation, the radial $\vec{E} \times \vec{B}$ drift and corresponding jet penetration.

\section{Jet motion outside the plasma}

Let us consider the gas jet, flowing out from the reservoir through the Laval nozzle with cylindrical cross-section and varying radius, see Fig. 4 . Let the $n_{r}, T_{r}$, and $p_{r}$ be the gas density, temperature and pressure in the reservoir respectively, while $n_{E}$ and $M_{E}$ are gas density and Mach number at the exit of the nozzle, and the nozzle radius at the exit is $r_{E}$. From the classical hydrodynamics [16]

$$
n_{E}=n_{r}\left(1+\frac{M_{E}^{2}}{2}\left(\gamma_{g a s}-1\right)\right)^{\frac{1}{1-\gamma_{g a s}}}=\frac{p_{r}}{T_{r}}\left(1+\frac{M_{E}^{2}}{2}\left(\gamma_{g a s}-1\right)\right)^{\frac{1}{1-\gamma_{g a s}}}
$$

where $\gamma_{\text {gas }}$ is a isentropic exponent.

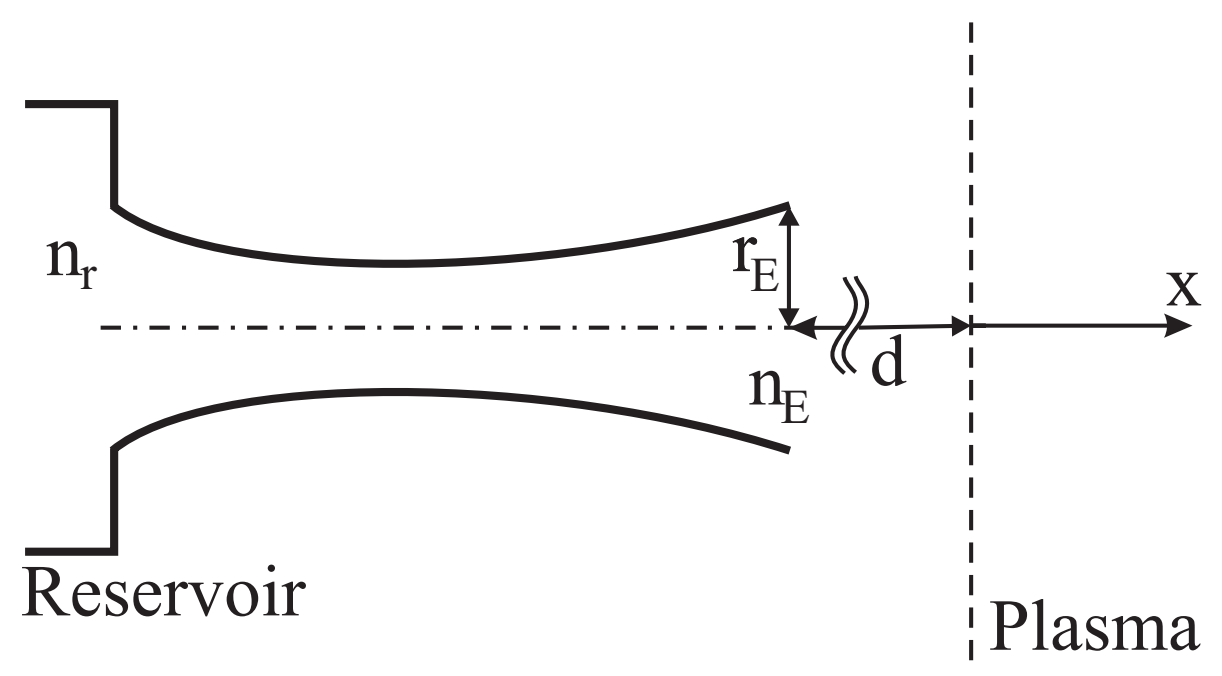

Figure 4. Laval nozzle.

Assuming that after release from the nozzle the gas expands in the perpendicular direction with sound velocity, one can estimate the jet cross-section at distance $d$ from the nozzle as $d=r_{E}\left(1+\frac{\widetilde{x}}{M}\right)$, where $\tilde{x}=x / r_{E}$. Therefore the density at distance $d$ is 


$$
n(d) \approx \frac{n_{E}}{\left(1+\frac{\tilde{x}}{M}\right)^{2}}=\frac{n_{r}}{\left(1+\frac{\tilde{x}}{M}\right)^{2}}\left(1+\frac{M_{E}^{2}}{2}\left(\gamma_{g a s}-1\right)\right)^{\frac{1}{1-\gamma_{g a s}}}=\frac{p_{r}}{T_{r}\left(1+\frac{\tilde{x}}{M}\right)^{2}}\left(1+\frac{M_{E}^{2}}{2}\left(\gamma_{g a s}-1\right)\right)^{\frac{1}{1-\gamma_{g a s}}}
$$

This expression might be used to estimate the jet density at the plasma.

\section{Model for jet heating and expansion along magnetic field lines}

Let us consider the 1D expansion of the partially ionized plasma confined in a tube with certain fixed cross-section, as shown in Fig. 5. Inside such a blob, where the collision frequency is high, and the mean free path of all species $\lambda_{e, i, a}$ is smaller than the jet parallel length $L, \lambda_{e, i, a}<<L$, the fluid equations are solved:

$$
\begin{aligned}
& \frac{\partial \rho}{\partial t}+\frac{\partial}{\partial z}\left(\rho V_{z}\right)=0 \\
& \rho \frac{\partial V_{z}}{\partial t}+\rho V_{z} \frac{\partial V_{z}}{\partial z}=-\frac{\partial p}{\partial z} \\
& \frac{3}{2} \frac{\partial p}{\partial t}+\frac{3}{2} \frac{\partial}{\partial z}\left(p V_{z}\right)=-\frac{\partial q_{i n c}}{\partial z}+\frac{\partial}{\partial z}\left(\chi \frac{\partial T}{\partial z}\right)-p \frac{\partial V_{z}}{\partial z}-Q_{\text {ioniz }_{2}}-Q_{\text {rad_losses }_{\text {_los }}}
\end{aligned}
$$

Here $\rho=M n_{h}$ is the mass density, $M$ is the atomic mass of jet atoms, $n_{i}, i=0 . . Z$ is the density of ions in the charge state $i, n_{h}=\sum_{i=0}^{Z} n_{i}$ is the total density of neutrals and atoms., $V_{z}$ is the parallel velocity, $p=\left(n_{e}+n_{h}\right) T$ is the total pressure, $T$ is the temperature common for all species, $n_{e}=\sum_{i=0}^{Z} i \cdot n_{i}$ is the electrons density, $q_{i n c}=q_{e h}+q_{i h}$ is the energy flux carried by the incident electrons $\left(q_{e h}\right)$ and ions $\left(q_{i h}\right)$ from the ambient plasma, $\chi$ is the heat conduction coefficient, $Q_{\text {ioniz }}$ and $Q_{\text {rad_losses }}$ are the ionization and radiation loss power per volume respectively.

The ionization state distribution is described by

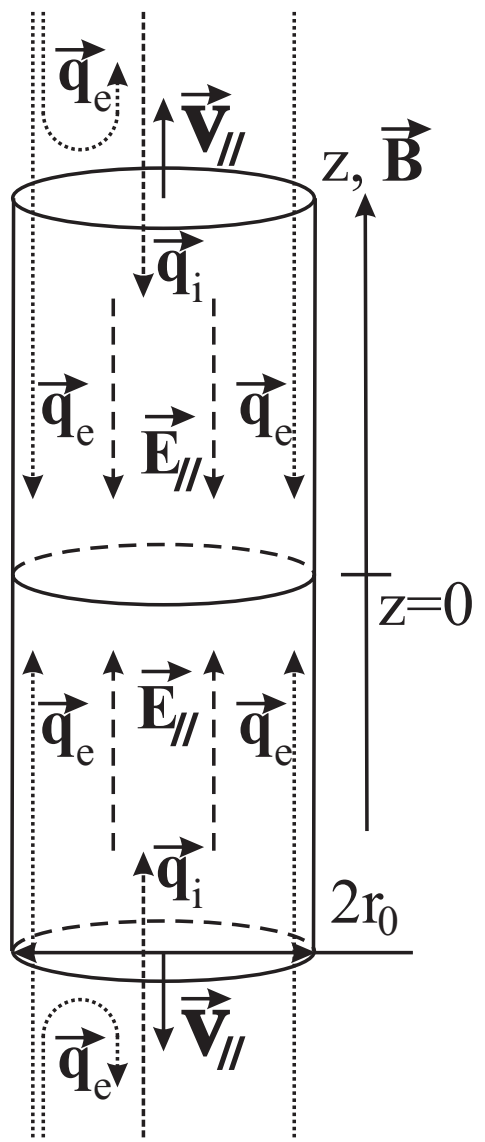

Figure 5.1D expansion of the jet along the magnetic field.

$$
\frac{d y_{i}}{d t}=n_{e}\left(y_{i+1} R_{i+1}-y_{i}\left(I_{i}+R_{i}\right)+y_{i-1} I_{i-1}\right)+n_{e}^{(i n c)}\left(y_{i-1} I_{i-1}^{(i n c)}-y_{i} I_{i}^{(i n c)}\right), i=0 . . Z
$$

where $y_{i}=n_{i} / n_{h}, I$ and $R$ are the ionization and recombination rates respectively, $y_{-1}=y_{Z+1}=0$, $R_{0}=I_{Z}=0, \frac{d}{d t}=\frac{\partial}{\partial t}+(\vec{V} \cdot \nabla)$. In the r.h.s. the first term represents, in particular, the ionization by cold 
electron impact, and the second term describes the impact by incident electrons penetrating through the gas jet from the ambient plasma.

The ionization/recombination rates and radiation losses are functions of density and temperature and are different for different conditions. For example, for an extremely dense deuterium jet one may use the Local Thermal Equilibrium approximation and assume surface radiation. On the contrary, for a weak impurity jet one may assume coronal dynamics. However, there is a wide range of parameters where these simple models are not valid, and one should use more sophisticated models (see for example [17]) or solve equations for the radiation transport, like it is done in [18]. In the Appendix such a model for Argon is presented. This model is used in the numerical simulations below. The coronal dynamics model is also used optionally to demonstrate the sensitivity of the results to the radiation loss model.

The incident electron and ion fluxes and corresponding energy deposition in the blob are calculated separately. First, since the mean free paths of ambient electrons and ions are large their deceleration inside the blob should be described kinetically. Second, fast electrons and ions carry the electric charge, therefore a self-consistent electric field should appear to provide quasi-neutrality.

The value of self-consistent electric field depends on the conductivity of the cold plasma. For a completely neutral blob the potential drop between it and the ambient plasma corresponds to the floating potential. In a Maxwellian ambient plasma with the density $n_{b g}$ and temperature $T_{e(b g)}$ the electron flux $\Gamma_{e h}^{(0)}=2 n_{b g} \sqrt{\frac{T_{e(b g)}}{2 \pi m_{e}}} \exp \left(-\frac{e \Delta \Phi}{T_{e(b g)}}\right)\left[1+\operatorname{erf}\left(\sqrt{\frac{e \Delta \Phi}{T_{e(b g)}}}\right)\right]^{-1}$

should be equal to the ion flux $\Gamma_{i h}=n_{b g} \sqrt{\frac{\gamma T_{e(b g)}}{m_{i}}}\left(m_{i}\right.$ is the ambient ion mass). From the condition $\Gamma_{e h}^{(0)}=\Gamma_{i h}$

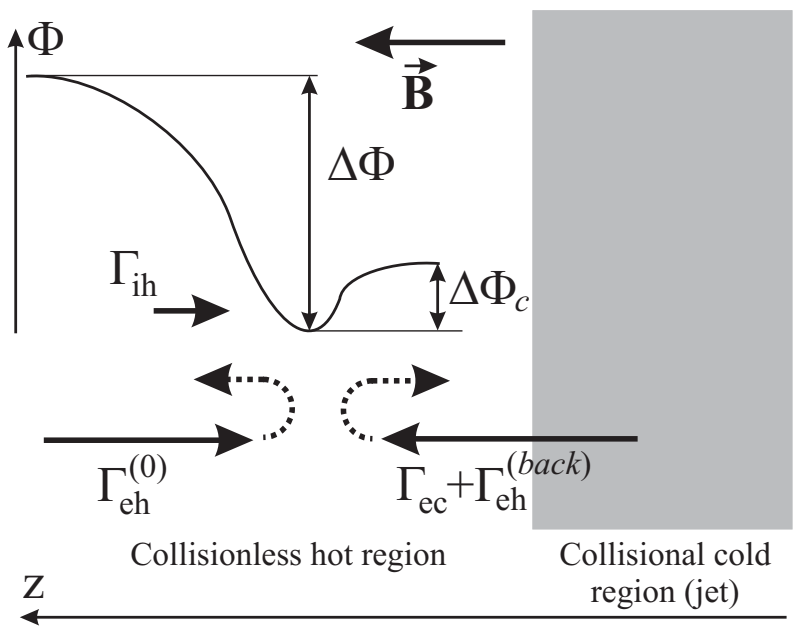

Figure 6. Double sheath formation. one obtains an equation for the magnitude of the potential drop $\Delta \Phi$ (see Ref [19] for details)

$$
\left[1+\operatorname{erf}\left(\sqrt{\frac{e \Delta \Phi}{T_{e(b g)}}}\right)\right] \cdot \exp \left(\frac{e \Delta \Phi}{T_{e(b g)}}\right)=2 \sqrt{\frac{m_{i}}{2 \pi \gamma m_{e}}}
$$

The solution of this equation is known as floating potential, $\Delta \Phi_{\text {float }} \approx 2.7 \frac{T_{e(b g)}}{e}$.

The situation is more complicated when the blob is partially ionized. In this case one should take into account the electrons extracted from the ambient plasma. The ambipolarity and quasi-neutrality conditions in the ambient plasma are $\Gamma_{e h}^{(0)}-\Gamma_{e c}=\Gamma_{i h}$ and $n_{e h}^{(0)}+n_{e c}=n_{i}=n_{b g}$, where subscripts $h, c$ correspond to the hot and cold electrons respectively. This problem of electrostatic shielding formation in the general case was studied in details in Ref [19], where equation for the potential drop was derived. In the case when the blob electron density is high enough (roughly when a maximal electron flux which could be extracted $\Gamma_{e c}=n_{e} \sqrt{\frac{T}{2 \pi m_{e}}}$ is larger than $\Gamma_{e h}^{(0)}$, see details in [19]), the double sheath is formed,

Fig. 6. The potential drop between the ambient plasma and the blob is smaller that the floating potential 
but still is of the order of $T_{e(b g)} / e$. This case might correspond to the real experiment parameters. Additional complication is connected with the possibility that some fast electrons might penetrate throughout the blob, forming a flux $\quad \Gamma_{e h}^{(\text {back })}$ from the cloud to the plasma, Fig. 6 . In this case one should add the corresponding terms in ambipolarity and quasi-neutrality conditions:

$$
\begin{gathered}
\Gamma_{e h}^{(0)}-\Gamma_{e c}-\Gamma_{e h}^{(b a c k)}=\Gamma_{i h}=n_{0} \sqrt{\frac{\gamma T_{e(b g)}}{m_{i}}} \\
n_{e h}^{(0)}+n_{e c}+n_{e h}^{(\text {back })}=n_{i}=n_{b g}
\end{gathered}
$$

The superscript 'back' shows that there are values corresponding to the electrons penetrating throughout the blob. These equations determine the potential drop between the ambient plasma and the blob used in the simulations.

Since the electrons stopping length inside the blob is larger than that of the ions for typical parameters of jet injection, the electron flux differs from the ion flux. To make the net current equal to zero inside the blob the electric field $E_{\|}$there should satisfy the equation

$$
0=e\left(\Gamma_{i h}-\Gamma_{e h}\right)+\sigma_{\|} E_{\|}-\sigma_{\|} \frac{T}{e}\left(g_{T} \frac{\partial}{\partial z} \ln T+\frac{\partial}{\partial z} \ln n_{e}\right)
$$

where $\sigma_{\|}$is the parallel conductivity, and $\Gamma_{e h}=\Gamma_{e h}^{(0)}-\Gamma_{e h}^{(b a c k)}$ Fig. 5. Note that since the conductivity is small the electric field becomes large enough to stop all the ambient electrons. In this case they are able to move only together with ions $\Gamma_{i h}=\Gamma_{e h}$. Eq. (5) for the potential distribution is also solved in the code.

The particle and energy flux depletion of hot background carriers (electrons and ions) inside the blob due to collisional interaction with the cold particles is modeled similarly to [20]. The distribution functions of the incident energy carriers (electrons and ions) are assumed to be Maxwellian and are modeled by 5 monoenergetic groups, carrying the equal energy flux. Hence the depletion of particle flux, energy and energy flux is calculated according to

$$
\begin{aligned}
& q_{k}=\sum_{j=1}^{5} \Gamma_{k}^{(j)} \varepsilon_{k}^{(j)}, k=e, i \\
& \frac{d \Gamma_{e}}{d z}=\Gamma_{e}\left(\frac{1}{\lambda_{e a}^{(m)}}+\frac{1}{\lambda_{e i}^{(m)}}+\frac{1}{\lambda_{e e}^{(m)}}\right) \\
& \frac{d \varepsilon_{e}}{d z}=\varepsilon_{e}\left(\frac{1}{\lambda_{e a}^{(\varepsilon)}}+\frac{1}{\lambda_{e i}^{(\varepsilon)}}+\frac{1}{\lambda_{e e}^{(\varepsilon)}}\right)-e E_{\|} \\
& \frac{d \varepsilon_{i}}{d z}=\varepsilon_{i}\left(\frac{1}{\lambda_{i B}^{(\varepsilon)}}+\frac{1}{\lambda_{i F}^{(\varepsilon)}}\right)+e E_{\|}
\end{aligned}
$$

Here $\varepsilon_{k}^{(j)}$ is the energy of each monoenergetic beam, $\lambda^{(m)}$ and $\lambda^{(e)}$ denote the mean free path lengths for momentum and energy transfer collisions, $(i B)$ and $(i F)$ subscripts correspond to ion-bound and ion-free electron collisions. The groups are introduced in such a manner that each group carries the same energy flux [20]. The expressions for the mean free paths in Eqs. (6)-(9) are taken from [21-25]. 

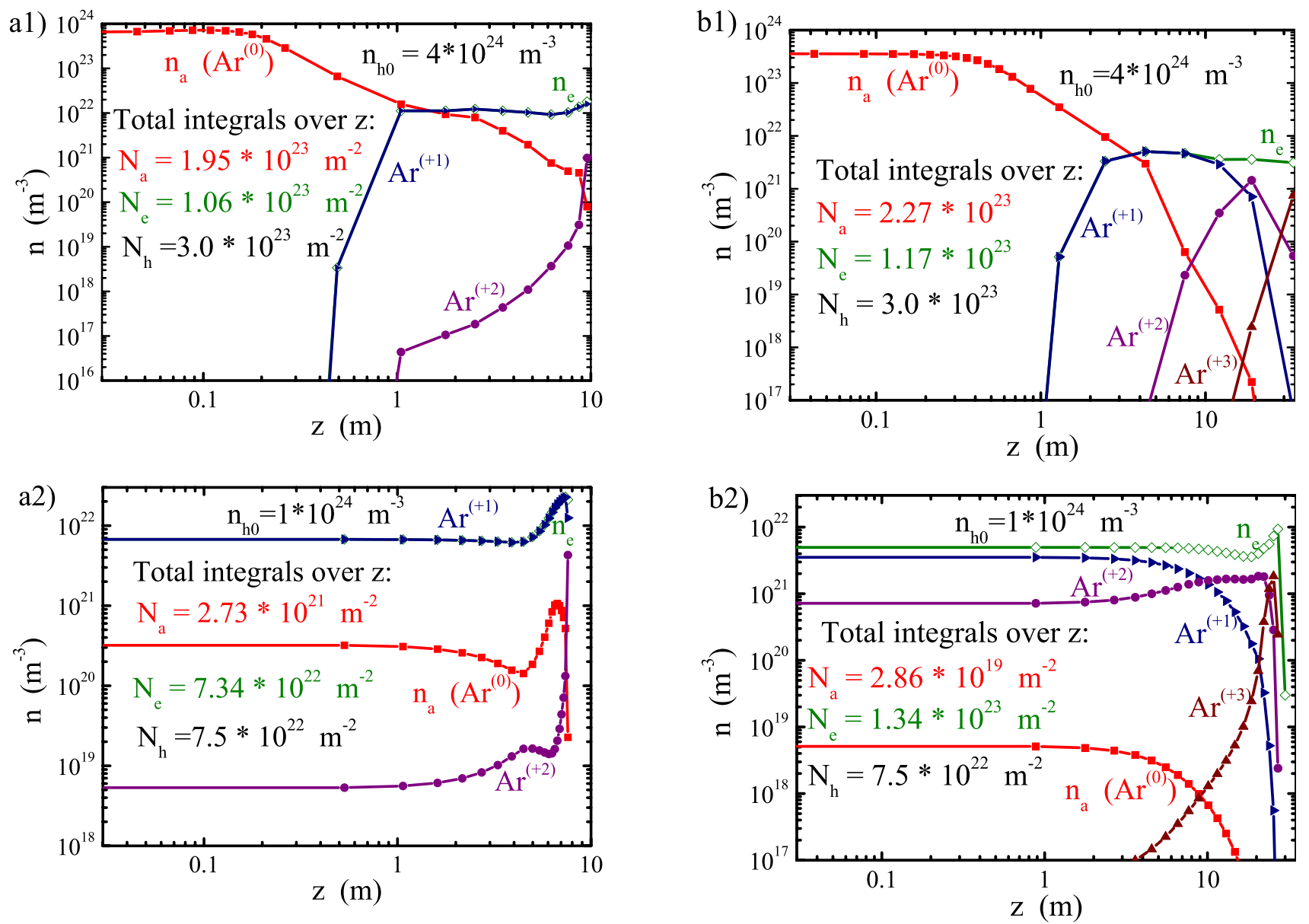

Figure 7. Density profile along the magnetic field. a1) - coronal dynamics model, initial density $\left.n_{h 0}=4 \cdot 10^{24} \mathrm{~m}^{-3} ; \mathrm{a} 2\right)$ - coronal dynamics model, initial density $n_{h 0}=1 \cdot 10^{24} \mathrm{~m}^{-3}$; b1) - model for optically thick blob, initial density $n_{h 0}=4 \cdot 10^{24} \mathrm{~m}^{-3}$; b2) - model for optically thick blob, initial density $n_{h 0}=1 \cdot 10^{24} \mathrm{~m}^{-3}$. Presented are integrals $N_{h}=\int_{0}^{\infty} n_{h} d z, N_{a}=\int_{0}^{\infty} n_{a} d z, N_{e}=\int_{0}^{\infty} n_{e} d z$

\section{Simulation of parallel expansion}

The approach described in the previous section was used to simulate the parallel expansion of the blob. The same set of equations has been previously solved in the pellet code LLP described in Ref. [11] to study the parallel expansion of the ablated material. In this section we consider evolution of an initially neutral blob along a magnetic field. The input parameters for modeling are ambient plasma temperature $T_{e(b g)}$ and density $n_{b g}$, blob initial density $n_{h 0}$ and temperature $T_{0}$, and also the initial length in z-direction $l_{0}$. The initial electrons density and parallel velocity $V_{z 0}$ are assumed to be zero. Presented below are results of simulation made for the parameters typical for the experiments with Argon jet injection on DIII-D. They are: $T_{e(b g)}=1.5 \mathrm{keV}$, $n_{b g}=3 \cdot 10^{19} \mathrm{~m}^{-3}, l_{0}=7.5 \mathrm{~cm}, n_{h 0}=4 \cdot 10^{24} \mathrm{~m}^{-3}$ and $n_{h 0}=1 \cdot 10^{24} \mathrm{~m}^{-3}$. In Figs 7-10 the main jet parameters at $t=1.5 \mathrm{~ms}$ are presented for two models for ionization state distributions and radiation losses and for different values of the initial density. 

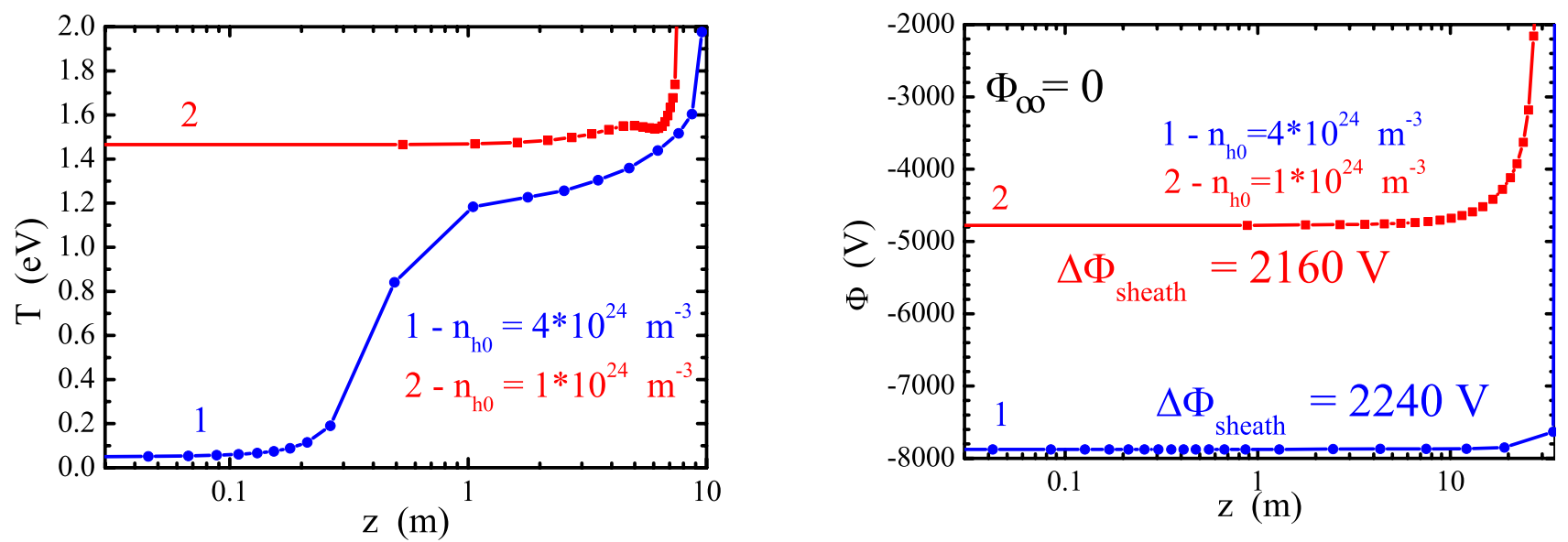

Figure 8. Temperature profile along the magnetic field. a) - coronal dynamics model; b) - model for optically thick blob.
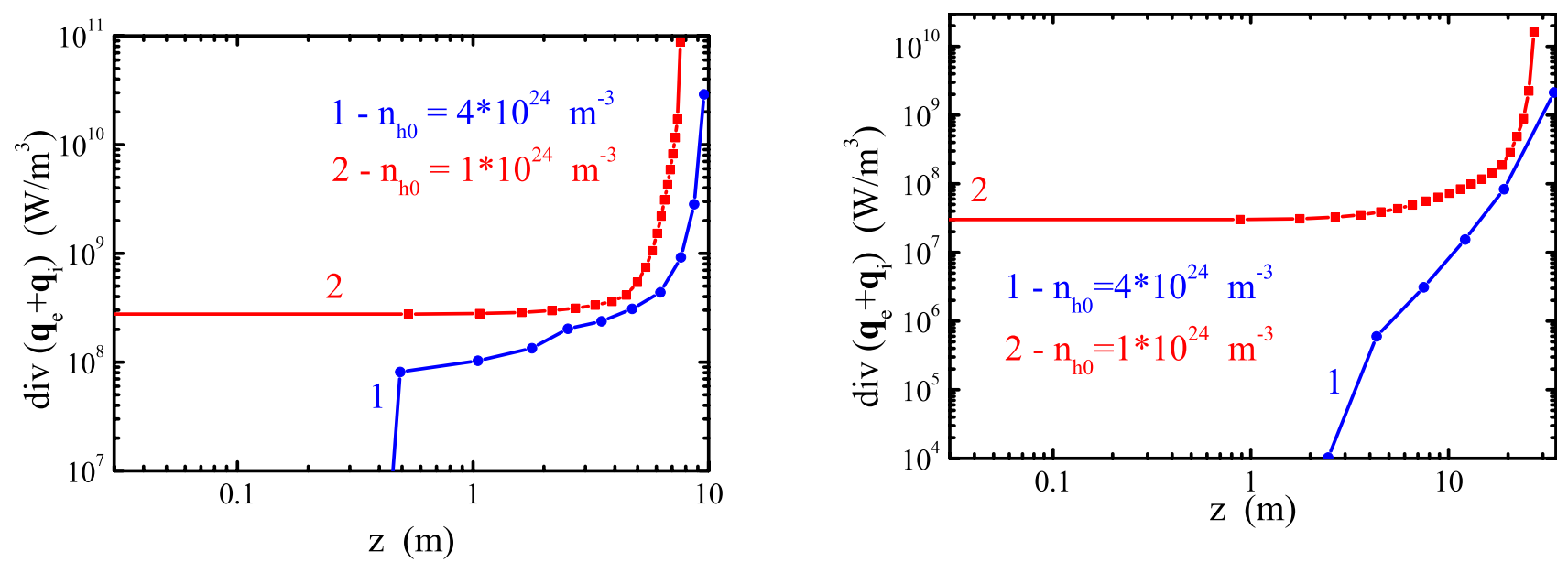

Figure 9. Heating power density due to incident particles deceleration. a) - coronal dynamics model; b) - model for optically thick blob.
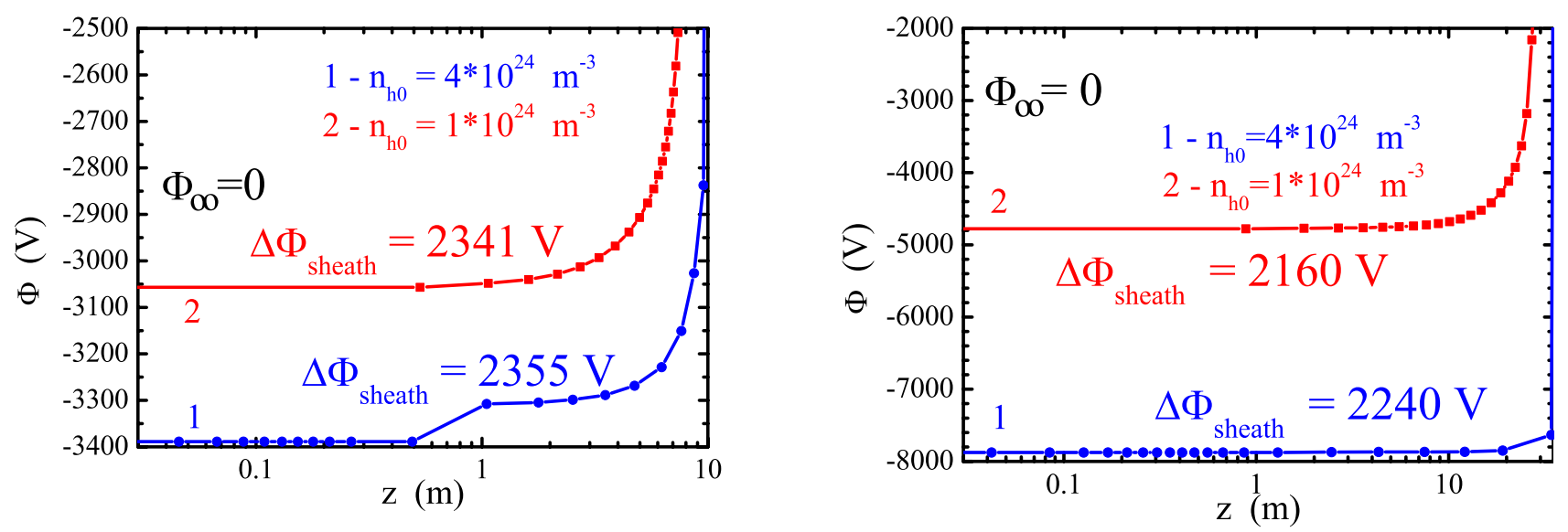

Figure 10. Electrostatic potential profile along the magnetic field. a) - coronal dynamics model; b) model for optically thick blob.

Two characteristic regions (which are schematically shown in Fig. 11) are clearly seen for the higher initial density independently of the model for ionization and radiation losses (see Figs. 7a1, 7b1 and curves marked by index 1, circles and blue colour in figures 8-10). The first region (I), at the outer part of the cloud, is a region where hot incident electrons are able to penetrate (i.e. where $\operatorname{div}\left(\vec{q}_{e}+\vec{q}_{i}\right)>0$, see 
Fig 9). On the density, temperature and electrostatic potential plots this region may be identified by the presence of ionized particles, the temperature values over $1 \mathrm{eV}$ and by the non-zero inclination of the potential curve correspondingly. Jet temperature in this region is defined mainly by the energy balance between the heating power coming from the incident particles and energy losses due to the radiation, therefore the radiation losses are almost the same for two models. The ionization degree (see Figs 7a1, 7b1), on the contrast, differs significantly, because the same amount of radiation in different models correspond to different temperatures and ionization state distributions. Note that the values of the density are lower than initial ones due to the free expansion along the magnetic field. One can see from Fig. 10 that the parallel electric field arises in the region I to provide a conductivity current of cold electrons, which is necessary to compensate the ambient electron flux according to Eq. (5). The second region (II) is a cold and dense one, to which hot background particles are not able to penetrate $\left(\operatorname{div}\left(\vec{q}_{e}+\vec{q}_{i}\right)=0\right)$. The temperature in this part of the cloud remains low (300 K approximately, see Fig. 8), and the electron density is close to zero (see Figs 7a1, 7b1), independently of the radiation losses model used. The electrostatic potential (Fig. 10) in this region is constant and the electric field is equal to zero because there are no electrons and ambient electrons do not penetrate there. It is also seen that the significant fraction of the total number of injected particles is located in region II.

Note that the net electrostatic potential drop (including both electrostatic sheath outside the cloud and the drop in region I, $\Delta \Phi_{\Sigma}$ ) is of the order of several $T_{e(b g)} / e$, i.e. enough to stop most part of the ambient electron flux.

For the smaller initial density of the blob the incident particles can penetrate up to its center, providing heating and ionization. Calculations show that for $n_{h 0}=1 \cdot 10^{24} \mathrm{~m}^{-3}$ and the same other parameters the incident particles can penetrate throughout the whole blob, and region II disappears (see Figs. 7a2, 7b2 and curves marked by index 2, squares and red colour in figures 8-10). The electron density becomes high enough everywhere in the blob.

For the fuelling jet, where the radiation losses are small compared to the heating power, the energy of the incident particles is spent mainly on heating, expansion and ionization. For the blob and background plasma parameters typical for the Tore Supra tokamak edge plasma $l_{0}=3 \mathrm{~cm}$, $n_{h 0}=4 \cdot 10^{21} \mathrm{~m}^{-3}, \quad T_{e(b g)}=100 \mathrm{eV}$, $n_{b g}=1 \cdot 10^{19} \mathrm{~m}^{-3}$ the ionization degree reaches unity rather fast. The computed density profiles corresponding to several moments are shown in Fig. 12.

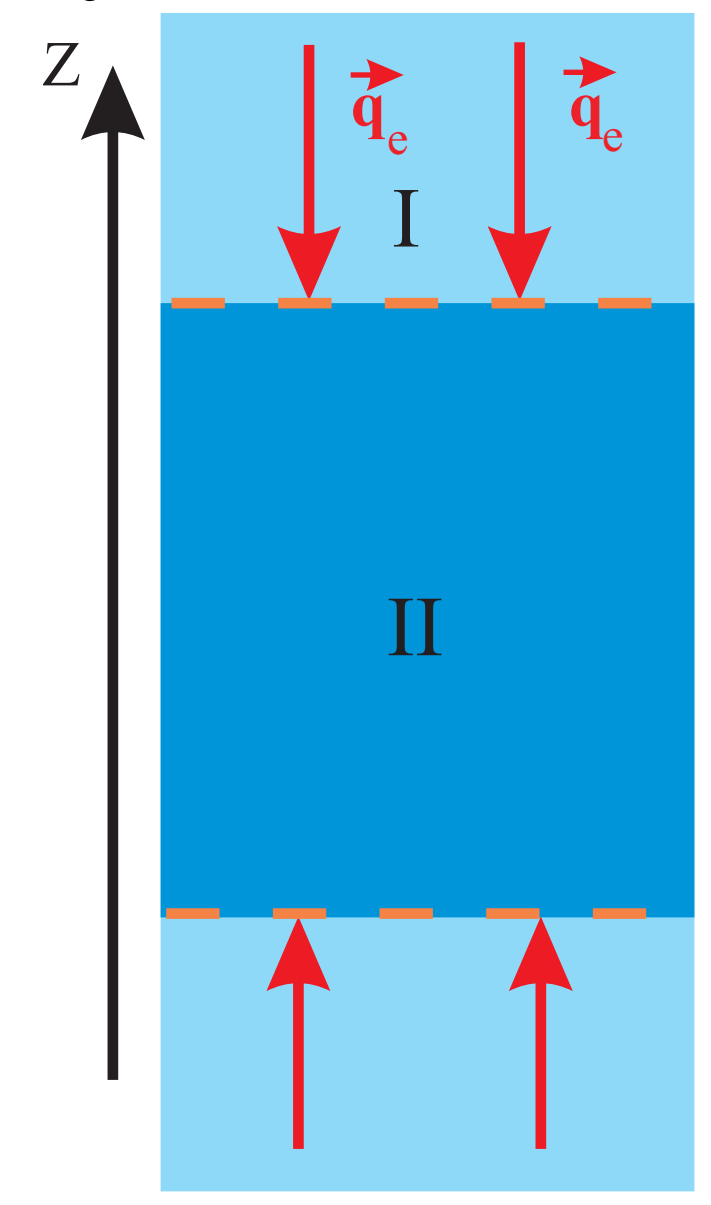

Figure 11. Schematic view of the expanding blob. Shown are the two qualitatively different regions, first one is the region where incident particles can penetrate and second is the region where they cannot penetrate. 


\section{Physics of expansion across magnetic field}

As it is known from the pellet physics, the cold dense (comparing with ambient plasma parameters) blob do not expands freely across the magnetic field due to the Lorenz force, balancing the pressure gradient [26]. The same may be expected also for the supersonic jet, and, as it is demonstrated below by running a $1.5 \mathrm{D}$ MHD code (similar to the LLP pellet code), that is the case.

In this section we study the conditions necessary to stop the expansion across the magnetic field in the slab geometry (for the simplicity). Consider a cold dense partially ionized blob with density $n_{h}$ inhomogeneous in the y direction. Density $n_{h}$ is assumed to be much larger than the ambient plasma density $n_{b g}$ while its temperature $T$ is supposed to be much smaller than the ambient temperature $T_{e(b g)}$. The ion density inside the blob $n_{i}$ is much smaller than the neutral atom density

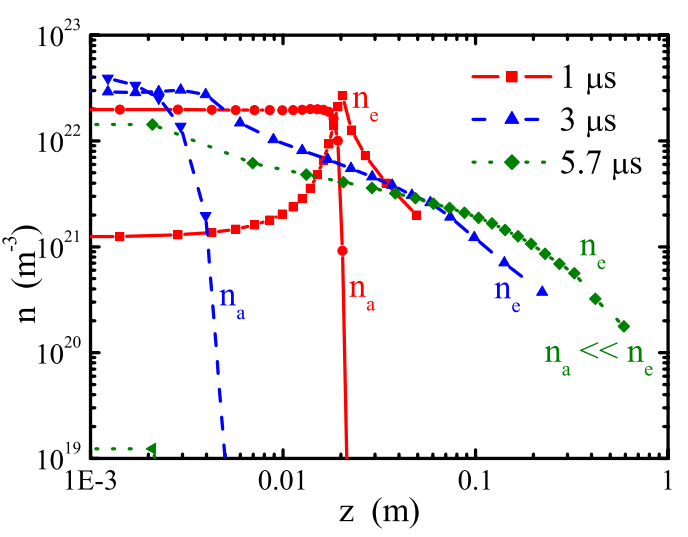

Figure 12. Density profile along the magnetic field $n_{b g}=1 \cdot 10^{19} \mathrm{~m}^{-3}$ at different times for the following set of deuterium blob and background plasma parameters:

$$
\begin{aligned}
& l_{0}=3 \mathrm{~cm}, n_{h 0}=4 \cdot 10^{21} \mathrm{~m}^{-3}, \\
& T_{e(b g)}=100 \mathrm{eV} .
\end{aligned}
$$
$n_{a}$ but larger than $n_{b g}: n_{b g}<<n_{i}<<n_{a}$.

Ions and neutrals of the blob are strongly coupled due to the high collision frequency so the common temperature is assumed. The magnetic field is sufficiently strong so that both blob and ambient pressures are significantly smaller than the magnetic pressure $B^{2} / 2 \mu_{0}$

Let us analyze the stage when the acceleration is finished and the force balance is achieved. The stage of expansion will be analyzed in the next section. The momentum balance in the $y$ directions is

$$
\frac{\partial p}{\partial y}=-j_{x} B, \quad,
$$

where $p=p_{a}+p_{i}+p_{e}$ is the sum of the neutral and plasma pressures of the blob. (Here and below subscript $e$ denotes electrons and subscript $i$ denotes ions). We assume that only single ionized ions are present. Expressing current through the magnetic field, we have standard pressure balance

$$
p+\frac{B^{2}}{2 \mu_{0}}=\frac{B_{0}^{2}}{2 \mu_{0}} .
$$

Note that the parameter $\beta=2 \mu_{0} p / B_{0}^{2}<<1$ is calculated using the full pressure of the blob, where the pressure of neutrals dominates. If the ionization degree is small, the perpendicular current in Eq. (10) is significantly larger than the diamagnetic current in fully ionized plasma. To understand this in more detail let us consider partial momentum balances.

For neutrals the friction with ions and electrons balances their pressure gradient 


$$
-\frac{\partial p_{a}}{\partial y}=n_{a} m_{a} v_{c x}\left(u_{a y}-u_{i y}\right)+n_{e} m_{e} v_{e a}\left(u_{a y}-u_{e y}\right), \quad v_{c x}=n_{i}\left\langle V \sigma_{c x}\right\rangle, \quad v_{e a}=n_{a}\left\langle V \sigma_{e a}\right\rangle
$$

where $\vec{u}_{a}$ is the velocity of neutral atoms, $\vec{u}_{e}$ is the velocity of electrons and is the ion velocity. The thermal force is neglected. For neutrals, according to Eq. (12), where friction with electrons may be neglected, the difference between neutral and ion velocities corresponds to the diffusive motion of neutrals through ions

$$
u_{a y}=-\frac{1}{n_{a} m_{a} v_{c x}} \frac{\partial p_{a}}{\partial y}+u_{i y}
$$

This relative neutral velocity is much smaller than the sound speed provided $\lambda_{a}=\left(n_{i} \sigma_{c x}\right)^{-1}<<L$, where $L$ is the spatial scale of the blob.

Neutral drag, Lorenz force and electric field balance the ion pressure:

$$
-\frac{\partial p_{i}}{\partial y}+e n_{i} E_{y}+n_{a} m_{a} v_{e x}\left(u_{a y}-u_{i y}\right)-e n_{i} u_{i x} B=0 .
$$

Electron-ion friction vanishes because $j_{y}=e n_{e}\left(u_{i y}-u_{e y}\right)=0$.

Since ion-neutral friction is equal to the neutral pressure gradient (see Eq. (12)), which could be several orders of magnitude larger than the plasma pressure gradient, the current in the $x$-direction is much larger than the ion diamagnetic current driven by the ion pressure gradient. The Lorenz force caused by the electron diamagnetic flux balances the electron pressure gradient, electron-neutral friction and electric field

$$
-\frac{\partial p_{e}}{\partial y}-e n_{i} E_{y}+e n_{i} u_{e x} B-n_{e} m_{e} v_{e a}\left(u_{e y}-u_{a y}\right)=0
$$

The sum of Eqs. (12), (14)-(15) gives Eq. (10).

Now let us find the electric field in the $y$-direction, $E_{y}$. Strong ion flux in the $x$ direction causes enhanced ion motion in the $y$ direction. Indeed, from the $x$ component of ion momentum balance we have

$$
e n_{i} u_{i y} B-n_{a} m_{a} v_{c x}\left(u_{i x}-u_{a x}\right)-n_{e} m_{e} v_{e i}\left(u_{i x}-u_{e x}\right)=0 .
$$

Summing this equation with $x$ component of electron momentum balance

$$
-e n_{e} u_{e y} B-n_{e} m_{e} v_{e a}\left(u_{e x}-u_{a x}\right)+n_{e} m_{e} v_{e i}\left(u_{i x}-u_{e x}\right)=0,
$$

taking into account the condition $j_{y}=0$, one finds

$$
-n_{a} m_{a} v_{c x}\left(u_{i x}-u_{a x}\right)-n_{e} m_{e} v_{e a}\left(u_{e x}-u_{a x}\right)=0 .
$$


Together with Eq. (10) in the form $n_{e}\left(u_{i x}-u_{e x}\right)=-\frac{1}{e B} \frac{\partial p}{\partial y}$, it yields

$$
u_{i x}=-\frac{1}{e n_{e} B} \frac{\partial p}{\partial y} \cdot \frac{n_{e} m_{e} v_{e a}}{n_{e} m_{e} v_{e a}+n_{a} m_{a} v_{c x}}+u_{a x}
$$

Substituting this into Eq. (14) with Eq. (13) one obtains the ambipolar electric field

$$
E_{y}=\frac{1}{e n_{e}} \frac{\partial\left(p_{i}+p_{a}\right)}{\partial y}-\frac{1}{e n_{e}} \frac{\partial p}{\partial y} \cdot \frac{n_{e} m_{e} v_{e a}}{n_{e} m_{e} v_{e a}+n_{a} m_{a} v_{c x}}
$$

The second term in this equation is small, because the electron-neutral friction is much smaller than the ion-neutral one. Consequently,

$$
E_{y}=\frac{1}{e n_{i}} \frac{\partial\left(p_{i}+p_{a}\right)}{\partial y}
$$

This electric field directed from the ambient plasma inside the jet is shown in Figs 2,3 by '+' signs. It is interesting to note that the ambipolar electrostatic potential could be much larger than the usual $T_{i} / e$ since the ratio $n_{a} / n_{i}>>1$. The ions do not move in the $\mathrm{x}$-direction, and the current in the $\mathrm{x}$-direction is carried by electrons: $j_{x}=e n_{e} u_{e x}=e n_{e} \frac{E_{y}}{B}$.

In summary we may conclude that the partially ionized plasma is confined by the magnetic field due to the interaction between magnetic field and perpendicular currents produced by the ionized fraction. In the case of supersonic jet this leads to the fact that the integral $\int_{-\infty}^{-\infty} n d z$ remains constant, which provides the incident energy carriers depletion and allows the jet to remain neutral and penetrate.

\section{Modeling of expansion across the magnetic field}

To obtain the poloidal size of the blob we consider a cigar-like structure and use the 1.5D MHD code (similar to the LLP pellet code). This is in fact the 2D code, where the equations along z-axis are averaged. In this code axial symmetry is assumed. The cloud is discretized in the radial $\left(B_{\perp}\right)$ direction, the axial $\left(B_{\|}\right)$expansion of each Lagrangian cell also being taken into account without discretization in that direction. The equations are single fluid MHD equations, same as are in $B_{\|}$code,

$$
\begin{aligned}
& \frac{\partial \rho}{\partial t}+(\nabla \cdot \rho \vec{V})=0, \\
& \rho \frac{\partial \mathbf{v}}{\partial t}+\rho(\vec{V} \cdot \nabla) \vec{V}=-\nabla p+[\vec{j} \times \vec{B}], \\
& \frac{3}{2} \frac{\partial p}{\partial t}+\frac{3}{2}(\nabla \cdot p \vec{V})=-\left(\nabla_{\|} \cdot \vec{q}_{i n c}\right)+\left(\nabla_{\perp} \cdot \chi \nabla_{\perp} T\right)-p(\nabla \cdot \vec{V})-Q_{\text {ioniz }_{2}}-Q_{\text {rad_losses }_{-}}+\frac{j^{2}}{\sigma},
\end{aligned}
$$


and equation (4) for charge state distribution. The input parameters are the ambient plasma temperature $T_{e(b g)}$ and density $n_{b g}$, initial tube transverse radius $r_{0}$, blob initial density $n_{h 0}$ and temperature $T_{0}$, and also the initial length in z-direction $l_{0}$. The initial electron density and velocities $V_{z 0}$ and $V_{r 0}$ are assumed to be zero.

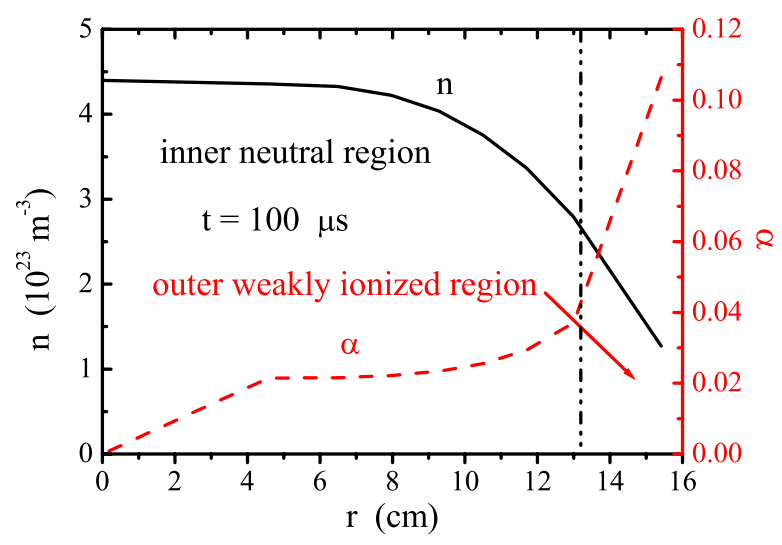

Figure 13. Typical radial density and ionization degree profiles.

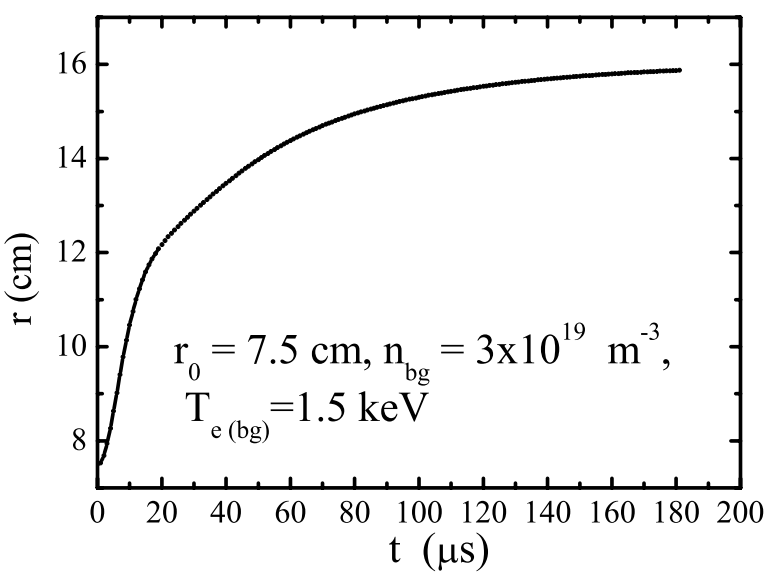

Figure 14. Time evolution of transverse size of the blob with the following parameters

$$
\begin{aligned}
& T_{e(b g)}=1.5 \mathrm{keV}, n_{b g}=3 \cdot 10^{19} \mathrm{~m}^{-3}, \\
& r_{0}=7.5 \mathrm{~cm}, n_{h 0}=4 \cdot 10^{24} \mathrm{~m}^{-3} .
\end{aligned}
$$

Calculations show that for wide ranges of parameters an ionization degree of the order of $5 \%$ establishes after several microseconds at the plasma periphery, where the density is smaller and where incident electrons can penetrate. The typical radial density and ionization degree profiles are presented in Fig. 13. The ionization degree of $5 \%$ is sufficient for the Lorenz force to stop the perpendicular motion. The time evolution profiles of the transverse size of the blob are presented in Fig 14. One can see that the transverse size of the blob does not change significantly (by a factor of two or less).

\section{Jet penetration in the radial direction}

According to the previous sections the jet expansion across the magnetic field is stopped by the diamagnetic current and corresponding $\vec{j} \times \vec{B}$ force. However, jet motion with a constant velocity in the radial direction is still possible due to the polarization electric field.

Let us consider the single cold dense partially ionized blob injected into the tokamak in the radial direction ( $x$-direction). The injected jet may be considered as a sequence of such blobs. The initial $(t=0)$ velocity both for neutral and ionized fraction of the blob is $V_{0}$. As shown above, the blob does not expand in poloidal direction (y direction). To provide motion of the blob ions in the $x$-direction the polarization electric field of dipole type $E_{0}=V_{0} B$ should appear, Fig. 3. Deceleration of ions in the $x$-direction is governed by the equation 


$$
m_{i} n_{i} \frac{d u_{i x}}{d t}=-j_{y} B-n_{0} m_{0} v_{c x}\left(u_{i x}-u_{0 x}\right)
$$

Here $j_{y}$ is additional to the current produced by the pressure gradient, Eq.(10), and in contrast to the diamagnetic current is not divergent free. If this current is not short-circuited in the ambient plasma, it should be zero. The ions of the blob hence are decelerated only due to the friction with neutrals. The friction with ions, on the other hand, determines the velocity of neutrals

$$
m_{0} n_{0} \frac{d u_{0 x}}{d t}=n_{0} m_{0} v_{e x}\left(u_{i x}-u_{0 x}\right)
$$

Since initial velocity both for ions and neutrals is $V_{0}$, the solution of Eqs. (22) and (23) corresponds to the constant velocity of the blob

$$
u_{i x}=u_{a x}=V_{0} .
$$

In the real 3D case, when the size of the jet along the magnetic field is finite, polarization field propagates along $\vec{B}$ with the Alfvén velocity. The current circuit is closed through the ambient plasma. As was demonstrated in [12], see also [14, 13], the ambient plasma in this case acts as an effective resistance with so-called wave conductivity $\sum_{W}=\frac{2}{\mu_{0} c_{A}}$, where $c_{A}$ is the Alfven velocity. Similarly to [12] one may substitute $j_{y}=\frac{m_{i} n_{i} u_{i x}}{B \tau_{i}}$ to the first term in r.h.s. of the Eq. (22), where $\left(u_{i x}=E_{y} / B\right)$

$$
\tau_{i}=\frac{\mu_{0} c_{A} M_{i}}{2 B^{2}}
$$

and $M_{i}, M_{N}$ are integral masses of the jet ions and neutrals

$$
M_{i, a}=\int_{-\infty}^{\infty} m_{i, a} n_{i, a} d z
$$

Eq. (22) yields

$$
m_{i} n_{i} \frac{d u_{i x}}{d t}=-m_{i} n_{i} \frac{u_{i x}}{\tau_{i}}-n_{i} m_{i} v_{i a}\left(u_{i x}-u_{a x}\right)
$$

with the ion-neutral friction force rewritten in the form $n_{a} m_{a} v_{c x}\left(u_{i x}-u_{a x}\right)=n_{i} m_{i} v_{i a}\left(u_{i x}-u_{a x}\right)$.

Let us estimate the two terms in Eq. (27) for parametres of the Argon jet on DIII-D. The Argon ion mobility with $n_{a}=2.7 \cdot 10^{25} \mathrm{~m}^{-3}$ is $b_{i}=1.52 \cdot 10^{-4} \frac{\mathrm{m}^{2}}{\mathrm{~V} \cdot \mathrm{s}}$ [27], consequently for the blob density $n_{a} \approx n_{h}=4 \cdot 10^{24} \mathrm{~m}^{-3}[5]$ the ion-neutral collision frequency is $v_{i a}=2.4 \cdot 10^{9} \mathrm{~s}^{-1}$. 
For the ambient plasma parameters $B=2.5 \mathrm{~T}, \quad n_{b g}=3 \cdot 10^{19} \mathrm{~m}^{-3}$ and $m_{b g}=3.34 \cdot 10^{-27} \mathrm{~kg}$ (the deuterium ambient plasma is considered) the Alfvén velocity is $c_{A}=\frac{B}{\sqrt{\mu_{0} n_{b g} m_{b g}}} \approx 7 \cdot 10^{6} \mathrm{~m} / \mathrm{s}$. The integrated jet mass $M_{i}$ may be estimated as $M_{i}=\alpha n_{h} m_{a} d$, where $d$ is the jet diameter and $\alpha=n_{i} / n_{h}$ is the ionization degree. Substituting $d=2 l_{0}=0.15 \mathrm{~m}$ [5] and estimating $\alpha=5 \%$, one finds $M_{i}=2 \cdot 10^{-3} \mathrm{~kg} / \mathrm{m}^{2}$. Thus the ion relaxation time is $\tau_{i}=1.4 \cdot 10^{-3} \mathrm{~s}$, and $\tau_{i}>>v_{i a}^{-1}$.

Thus for the typical Argon jet injected to DIII-D the neutral and ionized components are strongly coupled according to Eqs. (23), (27), i.e., $\left|u_{i x}-u_{a x}\right|<<\left|u_{a x}\right|$. Then integrating Eqs. (23), (27) over $z$ and summing them up with $u_{i x}=u_{a x}$, we find

$$
\frac{\partial E_{y}}{\partial t}=-\frac{E_{y}}{\tau_{W}}, \quad \tau_{W}=\frac{\mu_{0} c_{A}\left(M_{a}+M_{i}\right)}{2 B^{2}} .
$$

For the parameters of a Deuterium jet, the condition $\tau_{i}>>v_{i a}^{-1}$ is not fulfilled. Indeed, in analogy to previous estimates, the Deuterium ion mobility is $v_{i a}=6.1 \cdot 10^{6} \mathrm{~s}^{-1}$ for $n_{h}=4 \cdot 10^{21} \mathrm{~m}^{-3}$. For the ambient plasma parameters $B=2.5 \mathrm{~T}, \quad n_{b g}=3 \cdot 10^{19} \mathrm{~m}^{-3}, \quad d=0.12 \mathrm{~m} \quad$ and $\quad \alpha=0.2$, one finds $\tau_{i}=2.3 \cdot 10^{-7} \mathrm{~s} \sim v_{i a}^{-1}=1.6 \cdot 10^{-7} \mathrm{~s}$. Thus, the situation with a Deuterium jet is more complicated, because $\tau_{i}$ is smaller than the time necessary to reach the full ionization (see Fig. 12).

In the inhomogeneous magnetic field, the ionized plasma cloud in addition is accelerated in the low field side direction (LFS) [28]. This acceleration is $g=4 T / m_{i} R,\left(2 T=T_{e}+T_{i}\right)$, provided the Alfvén conductivity of the ambient plasma is neglected. For a partially ionized blob the acceleration is strongly reduced by a large factor $n_{h} / n_{i}$ since the $\nabla B$ drift current is proportional to the ion density while the acceleration of the blob contains the density of neutrals. Hence, the acceleration of the partially ionized blob is

$$
g=\frac{2\left(T_{e}+T_{i}\right)}{m_{i} R} \frac{n_{i}}{n_{h}} .
$$

Taking into account the Alfvén conductivity, the dynamics of the blob in the LFS direction is given by (see [13])

$$
\frac{\left(M_{a}+M_{i}\right)}{B^{2}} \frac{d E_{y}}{d t}=-2 \Sigma_{A}\left(E_{y}-E_{0}\right)+\frac{2 M_{i}\left(T_{e}+T_{i}\right)}{B R m_{i}} .
$$

Note that the ambient pressure $p_{b g}=2 n_{b g} T_{b g}=n_{b g}\left(T_{e(b g)}+T_{i(b g)}\right)$ does not appear in the Eq. (30). Therefore, the blob may penetrate inside the tokamak even if the ambient pressure $p_{b g}$ exceeds the pressure of the blob $p$. The ambient plasma simply moves together with the blob due to $\vec{E} \times \vec{B}$ drift 
since its density $n_{b g}$ is much smaller than the density of the blob $n_{h}$ and the electric field in the vicinity of the blob remains close to $E_{0}=V_{0} B$.

In the case of a fully ionized blob, i.e. when neutrals do not affect the ion dynamics, the result (30) is also valid. Including the wave conductivity, Eq. (22) transforms to Eq. (27) without ion-neutral friction and then to the Eq. (28), where one should treat $M_{a}<<M_{i}$ with $M_{a}+M_{i}=M_{h}$. Thus, the quantitative effect of the wave conductivity on the electric field relaxation is the same as for partially ionized plasma.

However, the acceleration caused by $\nabla B$ and curvature drift (29) in a fully ionized plasma is larger that in a partially ionized plasma, because the reduction factor $n_{h} / n_{i}$ is close to unity.

The equation (30) may be now rewritten in vector form

$$
\frac{\left(M_{a}+M_{i}\right)}{B^{2}} \frac{d \vec{u}_{x}}{d t}=-2 \Sigma_{A}\left(\vec{u}_{x}-\vec{V}_{0}\right)+\frac{2 M_{i}\left(T_{e}+T_{i}\right)}{B^{2} R^{2} m_{i}} \vec{R} .
$$

where the vector $\vec{R}$ is directed outward and its absolute value is equal to the tokamak major radius.

The blob dynamics (and, consequently, the jet behaviour) depends on the relation between $\tau_{W}$, $t_{g}=V_{0} / g$ and $t_{p e n}=a / V_{0}$ - the time interval necessary for the jet to reach the tokamak center moving with initial velocity $V_{0}$. If $\tau_{W}, t_{g} \gg t_{p e n}$, the blob (jet) may penetrate deep inside the tokamak moving with almost initial velocity. If $\tau_{W}, t_{g}<<t_{p e n}$, after $\tau_{W}$ the motion in the direction of $\vec{V}_{0}$ stops and after $t_{g}$ the motion turns into that with uniform velocity

$$
\vec{V}_{d}=\frac{M_{i}\left(T_{e}+T_{i}\right)}{B^{2} R^{2} m_{i} \Sigma_{A}} \vec{R}
$$

directed towards the LFS of the tokamak. Note that the drift velocity $V_{d}$ and integral $M_{i}$ are connected by the continuity equation $M_{i} V_{d} \delta=m_{i} n_{h 0} \pi r_{0}^{2} V_{0}$, where $\delta$ is the transverse size of the jet (see details in [13]). This size $\delta$ may decrease, because the drifting material moves along the converging equipotentials $[13,30]$, and increases due to diffusion. Estimating the jet penetration depth, one should take into account that $\nabla B$-induced current vanishes as the blob expands along the magnetic field due to the rotational transform (see details in [13]), consequently, the drifting blob stops after $t=R / c_{s}$.

If $\tau_{W}<<t_{p e n}, t_{g}$, after $\tau_{W}$ the blob comes to an almost full stop. In this case the penetration depth in this case may be roughly estimated as $V_{0} \tau_{W}$ (neglecting the diffusion).

\section{Modeling of the jet evolution}

To predict whether the jet is able to reach the tokamak center one should estimate $\tau_{W}$ and $t_{g}$ which depend on density, temperature and ionization degree. To calculate these parameters let us consider the jet as the sequence of blobs injected one after another with time interval $d t$. The initial parameters of each blob are the same: initial density $n_{h}$, initial velocity $V_{0}$ and initial longitudinal and radial sizes equal to each other, $r_{0}=l_{0}$. According to this approach the time interval between blob injections is set to 
$d t=r / V_{0}$. Then for each blob we may start the calculation of evolution along the magnetic field by means of the code described in Sections 3,4.

As the jet penetrates into a tokamak, the ambient plasma parameters are changed, and simultaneously is changed the transverse (poloidal) size. To take this into account, we start the evolution calculation with parameters corresponding to the plasma boundary and transverse size $r$ corresponding to these parameters calculated with help of 1.5D perpendicular code, described in Section 6. Then, as the first blob moves along its trajectory, the ambient density and temperature are increasing and a new transverse size $r$ is calculated. The time interval between the adjustment of the

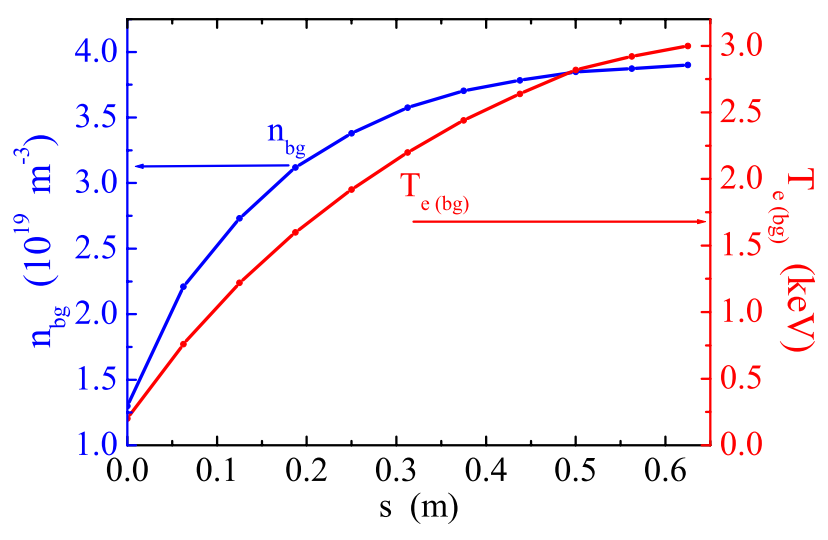

Figure 15. Typical density and temperature profiles used in the calculations for DIII-D. ambient plasma parameters is taken as $d t=r / V_{0}$.

To include the effect of an ambient plasma temperature drop after the injection, adiabatic cooling was assumed. For the next blobs evolution calculation we use the temperature value perturbed by all the previous blobs. This approach makes it possible to calculate in a self-consistent manner the temporal modification of the transverse size and corresponding change of the jet density as well as to trace the same background profiles in both calculation modules for each of the blobs. Note that the penetration velocity $V_{0}$ is the same for each blob and is constant, i.e. the polarization reduction effects considered in the Section 7 are not calculated.

Below presented are the results of calculations for the Argon jet injection for the disruption mitigation. The typical background temperature and density profiles used in calculations are presented in Fig. 15.

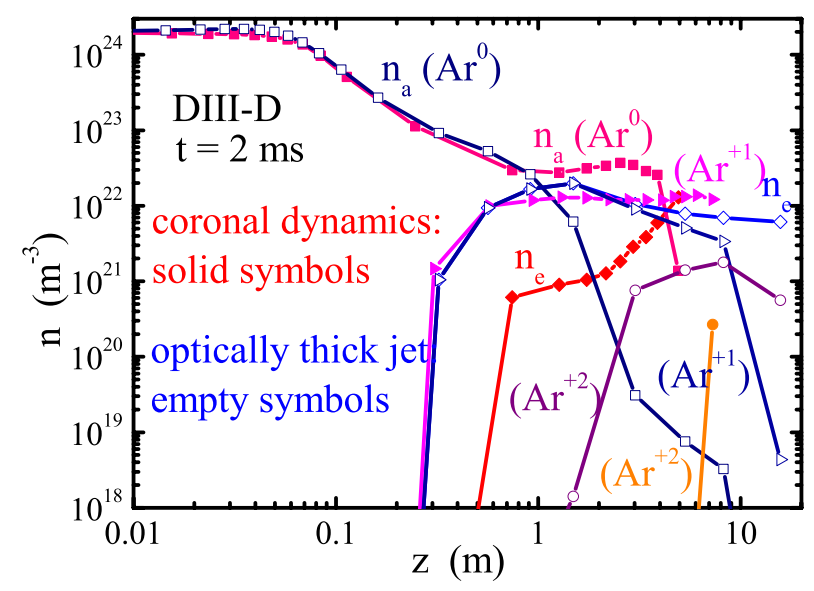

Figure 16 Density profiles along the magnetic field at $t=2 \mathrm{~ms}$.

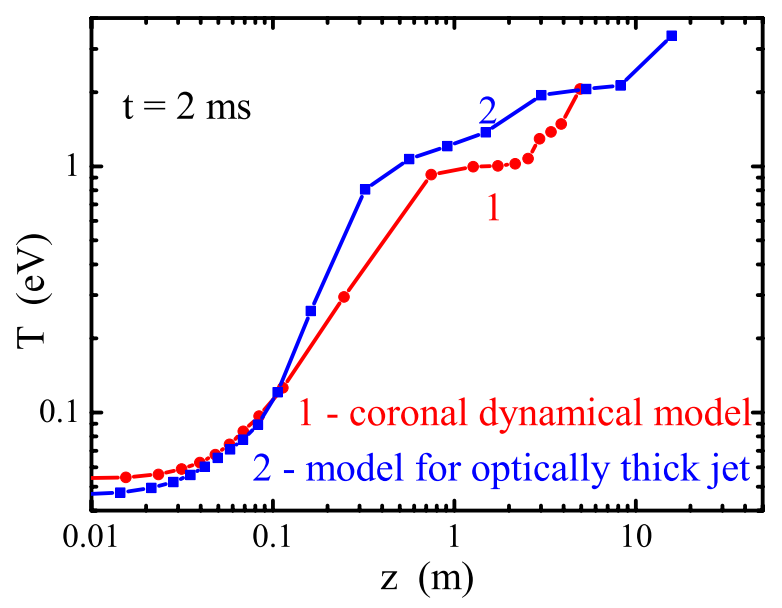

Figure 17. Temperature profile along the magnetic field at $t=2 \mathrm{~ms}$.

The initial jet parameters are the same as in Section 4: $r_{0}=7.5 \mathrm{~cm}, l_{0}=7.5 \mathrm{~cm}, n_{h 0}=4 \cdot 10^{24} \mathrm{~m}^{-3}$, $V_{0}=250 \mathrm{~m} / \mathrm{s}$. The time necessary for jet to reach the center thus is $2.5 \mathrm{~ms}$. In Figs. 16-17 the longitudinal density and temperature profiles (for the first blob, i.e. for the jet front) are presented for two radiation 
losses models at $t=2 \mathrm{~ms}$, i.e. at the four fifth of the distance to the tokamak center. These profiles are similar to those discussed in the Section 4: the most particles are located in the inner neutral region, where hot incident electrons cannot penetrate, and $\int n_{a} d z=2.13 \cdot 10^{23} \mathrm{~m}^{-2} \approx 0.7 \int n_{h} d z$.

The fraction of neutrals in the next blobs (in the jet body behind the front) is higher, because they are exposed to the ambient plasma with lower temperature. The temporal evolution of the ambient plasma temperature is shown in Fig 18. This figure demonstrates that only the energy losses on jet heating, expansion, ionization and radiation, but not the energy transport in the radial direction are taken into account. Also the width of the cooling front moving together with the jet cannot be found from such a simple analysis.

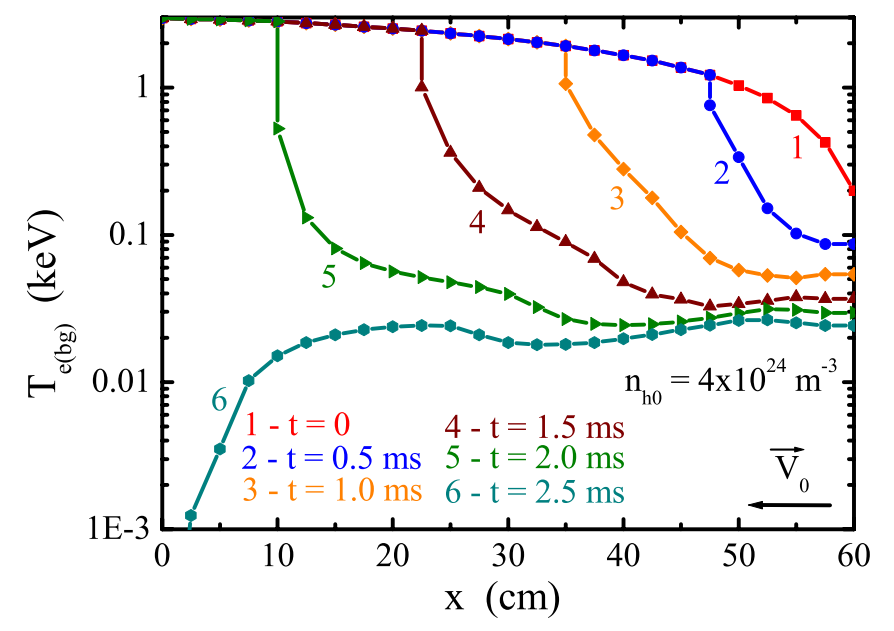

Figure 18. Temporal evolution of the ambient plasma temperature. cooling does not affect the penetration depth, which is defined by the density and temperature of the jet front, which is exposed to the unperturbed plasma.

Let us now estimate the characterizing time constants depending on the vertical polarization reduction $t_{g}$ and $\tau_{W}$. With $B=2.5 \mathrm{~T}, \quad \alpha=n_{i} / n_{h}=5 \%, \quad n_{b g}=3 \cdot 10^{19} \mathrm{~m}^{-3}, \quad n_{h 0}=4 \cdot 10^{24} \mathrm{~m}^{-3}$, $T_{i}=T_{e}=1 \mathrm{eV}, l_{z}=7.5 \mathrm{~cm}, R=1.65 \mathrm{~m}$ they are $\tau_{W}=28 \mathrm{~ms}$ and $t_{g}=0.9 \mathrm{~ms}$. One can see that for such a dense jet the Alfvén conductivity does not play a significant role, because $\tau_{W} \gg t_{p e n}$. On the contrary, the $\nabla B$-induced drift may stop the jet rather fast. However, the penetration depth cannot be found from such a simple $1 \mathrm{D}$ analysis, because the significant part of the jet remains neutral and insensitive to the electric field and because we do not consider the possibility for inner neutral and outer ionized parts of the cloud to move separately in the radial direction. This will be the aim of a future 3D analysis.

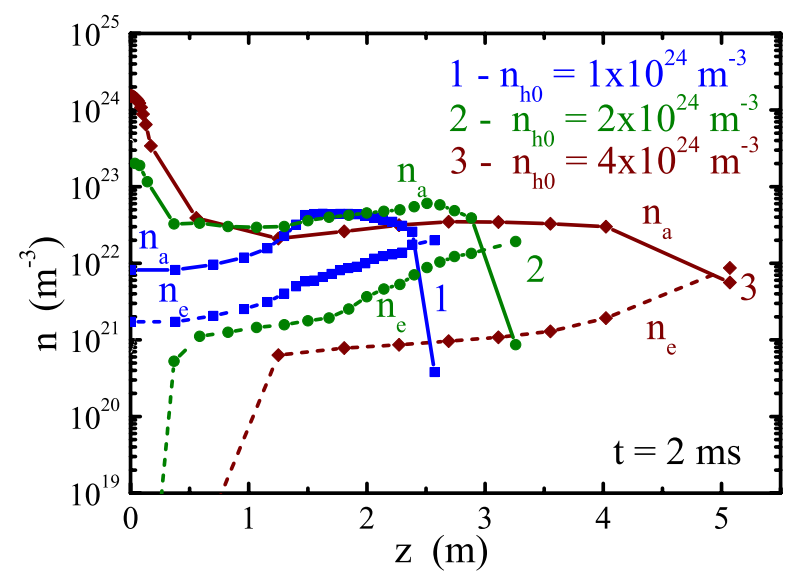

Figure 19. Longitudinal density profiles at $t=2 \mathrm{~ms}$ for the different values of the initial density.

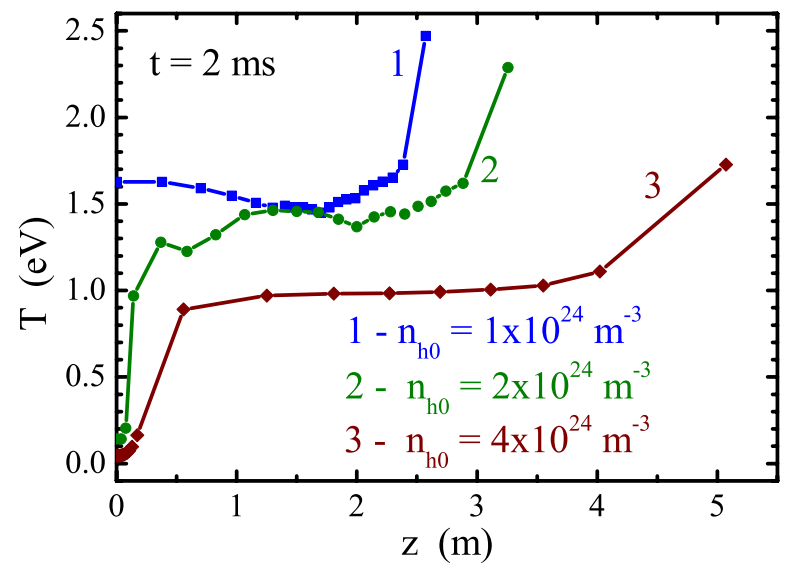

Figure 20. Longitudinal temperature profiles at $t=2 \mathrm{~ms}$ for the different values of the initial density. 
Nevertheless one can conclude that the jet may penetrate deep (up to about half a distance to the center) into a tokamak, because the significant part of the jet remains neutral.

The situation is opposite if one reduces the initial jet density (keeping the other parameters the same for the simplicity). In Figs 19-20 the longitudinal density and temperature profiles are presented at $t=2 \mathrm{~ms}$ for different values of the initial jet density $n_{h 0}$ (the coronal dynamics model for ionization state distribution and radiation losses is used). One can see that already for $n_{h 0}=1 \cdot 10^{24} \mathrm{~m}^{-3}$, the incident electrons penetrate throughout the whole jet, and the inner neutral cold region II disappears (see for comparison Section 4).

Thus, one can conclude that there is a critical value of the initial jet density $n_{c r}$. If $n_{h 0}>n_{c r}$, the incident electrons cannot reach the jet center, which remains cold, dense and neutral. Due to the large number of neutrals (insensitive to the electric and magnetic field) the jet may penetrate into the tokamak with its initial velocity. If $n_{h 0}<n_{c r}$, the jet is transparent for the incident electrons, and the ionization degree and temperature in the whole jet volume might be high enough to stop the jet propagation due to the $\nabla B$-induced drift. The critical density value depends on the tokamak plasma parameters and for the DIII-D tokamak is about $n_{c r}=(1 \div 2) \cdot 10^{24} \mathrm{~m}^{-3}$.

Therefore, one possible explanation why the jet did penetrate into the tokamak up to the half distance to the center in the first experiment [5] while it appeared only at the plasma periphery in the second one [7], would be that in the first experiment the jet density might exceeded the critical one, while in the second one the density might have been lower than the critical value.

Another mechanism, which might stop the jet, is that the fully ionized front of the jet in the inhomogeneous magnetic field acts as a piston, pushing the jet towards the LFS direction. The pushing force may be estimated as $\vec{F}=l_{x} l_{y} l_{z}[\vec{j} \times \vec{B}]$, where $l_{i}, i=x, y, z$, are the jet dimensions and $j=\frac{4 n_{\text {front }} T_{\text {front }}}{B R}$ is the vertical current caused by $\nabla B$-induced drift. The density in the jet front should be one order of magnitude smaller than the density of the jet itself $n_{h 0}<n_{c r}=(1 \div 2) \cdot 10^{24} \mathrm{~m}^{-3}$ to provide deep penetration of the ambient plasma energy carriers and full ionization. Therefore we may set $l_{x} l_{y} l_{z} n_{\text {front }}=0.1 \cdot d^{3} n_{h} \frac{l_{x}}{d}$, where $d$ is the initial jet diameter. The jet acceleration caused by this force may be found from the Newton's law $\widetilde{g}=F /\left(m_{i} n_{h} d^{2} a\right)=\frac{0.1 \cdot 4 \cdot T_{\text {front }} l_{x}}{m_{i} R a}$, where $a$ - the tokamak minor radius - is substituted for the jet length in $\mathrm{x}$ direction. Substituting, $T_{\text {front }}=1 \mathrm{eV}, R=1.65 \mathrm{~m}, a=0.60 \mathrm{~m}, l_{x}=1 \mathrm{~cm}$ one finds $\tilde{g}=9.6 \cdot 10^{3} \mathrm{~m} / \mathrm{s}^{2}$, and one gets as a time necessary to stop the jet $t_{\widetilde{g}}=\frac{V_{0}}{\widetilde{g}}=\frac{250 \mathrm{~m} / \mathrm{s}}{9.6 \cdot 10^{3} \mathrm{~m} / \mathrm{s}^{2}}=25.8 \mathrm{~ms}$. Note that the jet front size in the $x$ direction $l_{x}$ cannot be found rigorously from the analysis presented in this paper. If one estimates $l_{x} \sim d=0.15 \mathrm{~m}$, then with the same other parameters one finds $\tilde{g}=1.45 \cdot 10^{5} \mathrm{~m} / \mathrm{s}^{2}$ and $t_{\widetilde{g}}=1.7 \mathrm{~ms}$, which is less than $t_{p e n}=a / V_{0}$. Therefore, the effect discussed here might be important.

To obtain the deeper penetration with low density one needs to launch the jet from the high field side (HFS) of the tokamak. In this case the jet $\nabla B$ acceleration is directed towards the tokamak center and the jet front does not 'push' it outwards in a manner described above. 
The fuelling jet penetration was modeled with the parameters corresponding to the experiment [10] performed on ASDEX Upgrade. In this experiment the supersonic jet was suggested as a technique alternative to the injection of small pellets to cause ELM event. The result of the experiment was that such jet couldn't affect the ELM repetition rate in contrast to the pellets. This may be explained by the fact that such a jet launched from the LFS cannot penetrate inside the separatrix.

The jet parameters are [10] $l_{0}=r_{0}=6 \mathrm{~cm}, \quad n_{h}=1.7 \cdot 10^{21} \mathrm{~m}^{-3}$, $V_{0}=1800 \mathrm{~m} / \mathrm{s}$. The calculated density profiles corresponding to several time instants are demonstrated in Figure 21. It may be seen that the ionization degree reaches unity as the jet crosses the separatrix, in agreement with the experimental observation [10]. The explanation of this jet behaviour is the following. The background plasma temperature rises from several eVs in the SOL up to $100 \mathrm{eV}$ at several centimeters inside the separatrix. Consequently, since the radiation losses for Deuterium are negligible in comparison with the heating power due to incident electrons depletion, the jet temperature rises rather fast

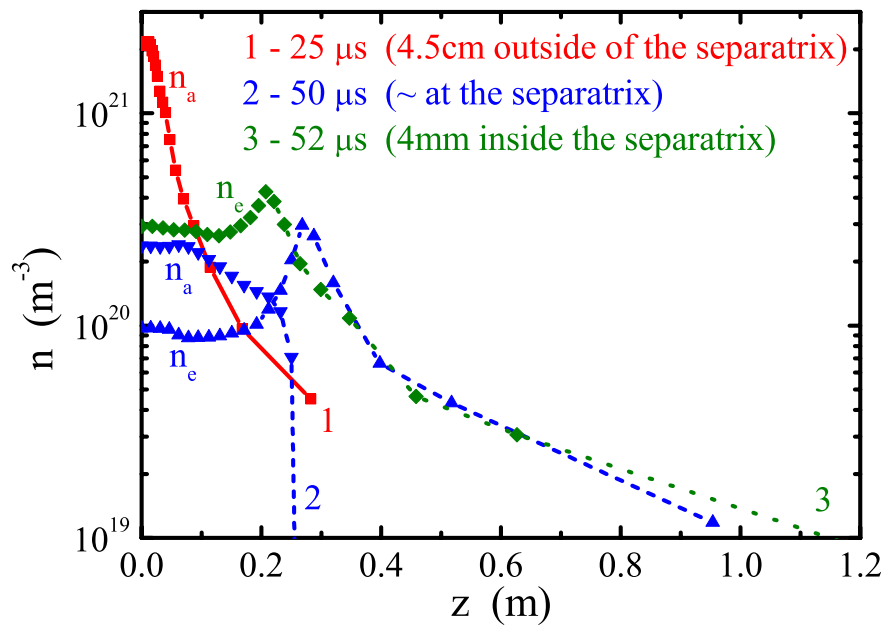

Figure 21. Longitudinal density profiles at different moments for the jet injected to ASDEX Upgrade [10]. and the jet becomes fully ionized within $1 \mu \mathrm{s}$.

With $B=2.5 \mathrm{~T}, \quad \alpha=n_{i} / n_{h}=1, \quad n_{b g}=5 \cdot 10^{18} \mathrm{~m}^{-3}, \quad n_{h}=1.7 \cdot 10^{21} \mathrm{~m}^{-3}, \quad T_{i}=T_{e}=10 \mathrm{eV}, \quad l_{z}=6 \mathrm{~cm}$, $R=1.65 \mathrm{~m}$ one finds $\tau_{W}=0.13 \mu \mathrm{s}$ and $\tau_{g}=1.6 \mu \mathrm{s}$. Consequently, as the front of the jet (launched from the LFS) becomes fully ionized it stops immediately and cannot penetrate further.

From the above estimates one can conclude that in the Tore Supra experiment [1], where the same experimental device was used, the jet behaved like a pure gas puff, i.e. the core fueling was provided by the diffusion mainly defined by a charge exchange processes. The moderate increase of fueling efficiency comparing with the gas puff may be due to the fact that particles of the jet are delivered directly to the separatrix (or last close flux surface, LCFS).

For the jet injected from the HFS there is one more mechanism which might provide a material delivery into the central regions of the plasma core - the $\nabla B$ induced drift. This drift causes the shift of the fully ionized part of the jet towards the LFS. The shift distance $\Delta x$ may be estimated in the manner

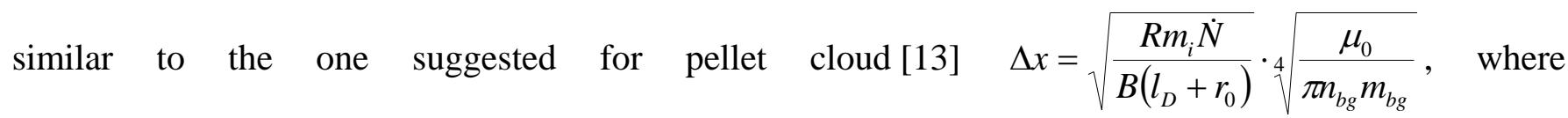
$l_{D}=\sqrt{4 D t}=\sqrt{\frac{4 D R}{C_{s}}}$ is the diffusive length, $D$ is the anomalous diffusion coefficient and the $\dot{N}=n_{h 0} \pi r_{0}{ }^{2} V_{0}$ is number of particles injected per second. For the parameters of Tore Supra experiment [1] the shift is negligible compared to the minor radius, $\Delta x \approx 5 \mathrm{~cm}$. This result agrees well with the fact that no significant increase of the fuelling efficiency with the injection from the HFS was observed [1]. Nevertheless, the $\nabla B$ induced drift seems to be the only mechanism, which might provide deep penetration of the fueling jet, therefore the HFS injection seems to be more promising. 


\section{Conclusions}

The evolution of the high-pressure supersonic gas jet injected into a tokamak plasma is studied. Since the jet expansion is similar to the expansion of the pellet ablation cloud, the LLP pellet code (with some modifications) was applied to model the jet expansion. The jet expansion, deceleration of the ambient electrons and ions by the jet, self-consistent electric field, elementary processes, radiation and adiabatic cooling of the ambient plasma are taken into account in the simulations. It is demonstrated that the ionization degree of the jet strongly depends on the jet parameters, especially on the initial jet density. For the high initial jet density the incident electrons cannot reach the jet center, which remains cold, dense and neutral. Due to the large number of neutrals the jet may penetrate into the tokamak with its initial velocity. For the lower initial jet density the jet is transparent for the incident electrons, and the ionization degree and temperature in the whole jet volume are high. For the DIII-D tokamak the critical density value is found to be of the order of $n_{c r}=(2 \div 3) \cdot 10^{24} \mathrm{~m}^{-3}$.

It is demonstrated that the small ionization degree is sufficient to stop the expansion across the magnetic field due to compensation of the neutrals pressure gradient by Lorenz force.

The jet propagates in the radial direction due to the poloidal electric field of dipole type. Two mechanisms of the reduction of this polarization (previously suggested for the pellet ablation cloud) are considered: the Alfvén conductivity of the ambient plasma and the $\nabla B$-induced drift. It is found that the first mechanism is not significant for the high-density jet. The $\nabla B$-induced acceleration towards the LFS in the case of partially ionized jet is reduced by factor of ionization degree with respect to the case of fully ionized plasma. If the jet density exceeds the critical one and the fraction of neutrals is large, the jet may penetrate deep inside the tokamak. Otherwise the ionization degree is high and the jet launched from the LFS should stop at the plasma periphery due to the $\nabla B$-induced drift. To obtain the deeper penetration of the low-density jet the recommendation is to launch it from the HFS.

The fueling jet with the parameters [1], [8-10] could not penetrate through the separatrix, because it is ionized in the separatrix vicinity and is stopped for the case of LFS injection. The jet injected from the HFS, on the contrary, after ionization may in principle penetrate towards the plasma center due to $\nabla B$ induced drift, however, for the parameters of experiment [1], the jet penetration depth is small compared to the tokamak minor radius.

\section{Acknowledgements}

Authors are grateful to the international ITER team and especially to Dr V. Mukhovatov for the support of this work.

\section{References}

[1] J.Bucalossi et al, Proceedings of $29^{\text {th }}$ EPS Conference on Contr. Fusion, Montreaux, 2002, ECA Vol. 26B, O-2.07 (2002)

[2] J.Bucalossi et al, in Fusion Energy 2002 (Proc. 19th Int. Conf. Lyon, 2002) (Vienna: IAEA) CDROM fileEX/P4-04, http://www.iaea.org/programmes/ripc/physics/fec2002/html/fec2002.htm

[3] J. Li et al, Plasma Phys. and Control. Fusion, 42 (2000) 135.

[4] L. Yao et al.,Nuclear Fusion, 44 (2004) 420

[5] D.G. Whyte et al. Journal of Nuclear Materials 313-316 (2003) 1239 
[6] D.G. Whyte et al. Phys. Rev. Letters 89, (2002) 055001

[7] E. Hollmann et al. in Fusion Energy 2004 (Proc. 20th Int. Conf. Vilamoura, 2004) (Vienna: IAEA)

CD-ROM file EX/10-6, http:/www-naweb.iaea.org/napc/physics/fec/fec2004/datasets/index.html

[8] J.Bucalossi et al, Proceedings of $31^{\text {th }}$ EPS Conference on Plasma Physics, London, 2004, ECA Vol.

28G, P-4.115 (2004)

[9] P.T. Lang et al. Nucl. Fusion 45 (2005), 502-511.

[10] P T Lang et al. Plasma Physics and Controlled Fusion 47 (2005), 1495-1516.

[11] L.L. Lengyel et al, Nucl. Fusion 39, 791 (1999).

[12] M. Scholer, Planet. Space Sci. 18, (1980) 977

[13] V. Rozhansky et al., Plasma Phys. and Control. Fusion 46 (2004) 575-591

[14] P.B. Parks et al., Phys. Plasmas 7, (2000) 1968

[15] P. Parks et al., Fusion Science and Technology, Vol. 35 (1999), 267.

[16] L. Landau, E. Lifshitz, Fluid Mechanics (Course of Theoretical Physics, Vol.6), BUTTERWORTH HEINEMANN, Oxford, 1997

(http://www.elsevier.com/wps/find/bookdescription.cws_home/676779/description\#description)

[17] D. Kh. Morozov et al, Nuclear Fusion 44 (2004) 252

[18] Karlykhanov, N.G., Martynenko, Yu.V. Matvienko, Yu.I., Moskovkin, P.G., Politov, V.Yu., Plasma Phys. Rep., 22 (1996) 903.

[19] Lengyel L.L., Rozhanskij V.A., Veselova I.Yu., Nucl. Fusion 36 (1996) 1679.

[20] W.A. Houlberg, et al, Nucl. Fusion 28 (1988) 595.

[21] Katz, L., Penfold, A.S., Rev. Mod. Phys. 21 (1952) 28.

[22] Parks, P.B., Leffler J.S., Fisher, R.K., Nucl. Fusion 28 (1988) 477.

[23] Huba, J.D., Collisions and Transport, 1994 Revised NRL Plasma Formulary, Nuclear Regulatory

Commission, Washington, DC (1994).

[24] Mehlhorn, T.A., J. Appl. Phys. 52 (1981) 6522.

[25] Linhard, J., Phys. Rev. 124 (1961) 128.

[26] Lengyel L.L., Spathis P.N., Nuclear Fusion 34, (1994) 675

[27] H.W. Ellis et al., Atomic Data and Nuclear Data Tables 17 (1976) 177; 22 (1978) 179

[28] Veselova I. Yu. and Rozhansky V.A., Sov. J. Plasma Phys 17 (1991) 817

[29] B.V. Kuteev, Fusion Technol. 26, 938 (1994)

[30] V.Rozhansky, I. Veselova, and S. Voskoboynikov, Plasma Phys. Control. Fusion 37, 399 (1995).

[31] W. Lotz, Astrophys. Journ. Suppl (1967), v. 14, p. 207

[32] Gervids, V.I., Zhidkov, AG., Marchenko, V.S., and Yakovlenko, S.I., in Reviews of Plasma Physics,

Ed. by M.A. Leontovich and B.B. Kadomtsev (Energoatomizdat, Moscow, 1982; Consultants Bureau, New York, 1987), vol. 12, p. 207.

[33] Encyclopedia of Low Temperature Plasma, Ed by V.E. Fortov, Introduction Volume, page I-223 MAIK, Nauka/Interperiodika, Moscow, 2000 (in Russian)

[34] D.A. Verner, E.M. Verner and G.J. Ferland, Atomic Data and Nuclear Data Tables, vol. 64 (1996) 1180 


\section{Appendix. Ionization and recombination rates and radiation losses in optically thick Argon cloud}

Ionization and recombination rates and radiation losses are calculated in a way similar to one presented in [17] with account of ionization from excited levels, de-excitation by electron impact and effective increasing of the lifetime of excited ion due to reabsorbtion and re-radiation of photon.

\section{Ionization}

Ionization by cold electron impact from the ground level is [31,32]

$$
I_{i}^{(g r)}=\frac{K_{i} \cdot \operatorname{Ei}\left(E_{i} / T\right)}{\sqrt{T}}
$$

Here $\operatorname{Ei}(x)=\int_{x}^{\infty} \frac{e^{-t}}{t} d t$, and $E_{i}$ are the ionization energies (in $\mathrm{eV}$ ) for the ion in the charge state $i$.

$T$ is also in $\mathrm{eV}$. The ionization energies $E_{i}$ and coefficients $K_{i}$ are specified in the Table 1

Table 1.

\begin{tabular}{|c|c|c|}
\hline$i$ & $E_{i}, \mathrm{eV}$ & $K_{i}, 10^{-13} \mathrm{~m}^{3} \cdot \mathrm{eV}^{1 / 2} / \mathrm{s}$ \\
\hline 0 & 15.76 & 1.7 \\
\hline 1 & 27.63 & 0.97 \\
\hline 2 & 40.91 & 0.655 \\
\hline 3 & 59.81 & 0.447 \\
\hline 4 & 75.0 & 0.357 \\
\hline 5 & 91.01 & 0.294 \\
\hline 6 & 124.32 & 0.215 \\
\hline 7 & 143.4 & 0.187 \\
\hline 8 & 422.04 & 0.0 .063 \\
\hline 9 & 478.7 & 0.056 \\
\hline 10 & 539.0 & 0.050 \\
\hline 11 & 618.3 & 0.043 \\
\hline 12 & 686.3 & 0.039 \\
\hline 13 & 755.7 & 0.0354 \\
\hline 14 & 854.8 & 0.0314 \\
\hline 15 & 918.0 & 0.0292 \\
\hline 16 & 4120.7 & 0.0065 \\
\hline 17 & 4426.2 & 0.00605 \\
\hline
\end{tabular}

\section{Ionization from excited levels}

Ionization by cold electron impact from excited levels is [33] 


$$
I_{i}^{(e x c)}=\frac{2 C}{(2 \pi)^{3 / 2}} \frac{g_{i+1}}{g_{i}} \frac{m_{e} e^{10}}{\hbar^{3} T^{3}} \exp \left(-\frac{E_{i}}{T}\right) \mathrm{cm}^{3} \cdot \mathrm{s}^{-1}
$$

where $e=4.8 \cdot 10^{-10}$ units CGS is the electron charge, $g_{i}$ are the statistical weights and $C$ is a constant with dimension: $C=2.12 \pm 1.1$. All quantities in this expression are in CGS units.

Setting $\frac{g_{i+1}}{g_{i}} \approx 1$, one obtains

$$
I_{i}^{(e x c)}=\frac{3.27 \cdot 10^{-11}}{(T(e V))^{3}} \exp \left(-\frac{E_{i}}{T}\right) \mathrm{m}^{3} \cdot \mathrm{s}^{-1}
$$

This expression gives the same results as Lotz's formulas [31], but is much simpler for calculations.

Thus, ionization by cold electron impact is

$$
I_{i}=I_{i}^{(g r)}+I_{i}^{(e x c)}
$$




\section{Photorecombination}

Expressions for photorecombination are following

$$
\begin{gathered}
R_{i}^{(p h)}=2.6 \cdot 10^{-20}\left(\alpha_{1}+\alpha_{2}\right) \mathrm{m}^{3} / \mathrm{s} \\
\alpha_{1}=i^{2}(R y / T)^{1 / 2} \frac{\mu}{n^{3}} \frac{E_{i-1}}{T} \exp \left(E_{i-1} / T\right) \cdot \operatorname{Ei}\left(E_{i-1} / T\right) \\
\alpha_{2}=2 i \sum_{v=1}^{\infty}\left(\frac{R y \cdot i^{2}}{(n+v)^{2} T}\right)^{\frac{3}{2}} \cdot \exp \left(\frac{R y \cdot i^{2}}{(n+v)^{2} T}\right) \cdot \operatorname{Ei}\left(\frac{R y \cdot i^{2}}{(n+v)^{2} T}\right)
\end{gathered}
$$

where $T$ is in $\mathrm{eV}, R y=13.6$ is the Rydberg constant in $\mathrm{eV}, n$ is the main quantum number, $\mu$ is the number of free electrons in the shell, which are listed in the Table 2.

Table 2.

\begin{tabular}{|c|c|c|}
\hline$i$ & $n$ & $\mu$ \\
\hline 0 & 1 & 0 \\
\hline 1 & 3 & 11 \\
\hline 2 & 3 & 12 \\
\hline 3 & 3 & 13 \\
\hline 4 & 3 & 14 \\
\hline 5 & 3 & 15 \\
\hline 6 & 3 & 16 \\
\hline 7 & 3 & 17 \\
\hline 8 & 3 & 18 \\
\hline 9 & 2 & 1 \\
\hline 10 & 2 & 2 \\
\hline 11 & 2 & 3 \\
\hline 12 & 2 & 4 \\
\hline 13 & 2 & 5 \\
\hline 14 & 2 & 6 \\
\hline 15 & 2 & 7 \\
\hline 16 & 2 & 8 \\
\hline 17 & 1 & 1 \\
\hline 18 & 1 & 2 \\
\hline
\end{tabular}

It is recommended to leave only three first terms in the sum in the expression for $\alpha_{2}$ 


\section{Dielectronic recombination}

The dielectronic recombination rate may be calculated as

$$
R_{i}^{(\text {diel })}=B_{i} \sum_{j=1}^{3} A_{i j} D_{i j} \frac{\exp \left(-E_{i j}^{(t r)} / T\right)}{T^{3 / 2}}
$$

Where $T$ is in $\mathrm{eV}$,

$$
\begin{gathered}
B_{i}=6.5 \cdot 10^{-16}(i+1)^{2} \sqrt{\frac{i}{i^{2}+R y}} \mathrm{~m}^{3} / \mathrm{s} \\
A_{i j}=\frac{f_{i j}^{(t r)} \sqrt{E_{i j}^{(t r)}}}{1+0.105 a_{i j}+0.015 a_{i j}{ }^{2}}, \text { if } \Delta n_{i j}=0 \\
A_{i j}=\frac{0.5 f_{i j}^{(t r)} \sqrt{E_{i j}^{(t r)}}}{1+0.21 a_{i j}+0.03 a_{i j}^{2}}, \text { if } \Delta n_{i j} \neq 0 \\
D_{i j}=E_{i j}^{(t r)} /((i+1) R y) \\
D_{i j}=\frac{0.0015\left((i+1) n_{t}\right)^{2}}{1+n_{t} / 200}, \text { if } \Delta n_{i j}=0 \\
n_{t}=\left(\frac{4.0015\left((i+1) n_{t}\right)^{2}}{\sqrt{1000}} i^{6} \frac{\sqrt{T(e V)}}{n_{e}} n_{i j} \neq 0\right.
\end{gathered}
$$

Where $E_{i j}^{(t r)}$ are the energies of the excited level, $f_{i j}^{(t r)}$ are the oscillator strengths, $\Delta n_{i j}$ are the changes of the main quantum number for the transition from level $j$ to the ground level, $R y=13.6$ is the Rydberg constant in $\mathrm{eV}$. The values $E_{i j}^{(t r)}, f_{i j}^{(t r)}$ and $\Delta n_{i j}$ are specified below in Table 3.

Thus, dielectronic recombination rate is

$$
R_{i}^{(\text {diel })}=\sum_{j=1}^{3} C_{i j}^{(d i e l)} D_{i j} \frac{\exp \left(-E_{i j}^{(t r)} / T\right)}{T^{3 / 2}}
$$

where $C_{i j}^{(d i e l)}$ is specified in the Table 3. Note that only 3 first level should be taken into account, if there are any. 


\section{Three-body recombination}

The three-body recombination rate

$$
R_{i}^{(3 b)}=8.75 \cdot 10^{-39} \cdot i^{3} \cdot n_{e} \cdot T^{-9 / 2} \mathrm{~m}^{3} / \mathrm{s}
$$

is calculated only for 4 first ions $(i<4)$. Here $n_{e}$ is in reversed cubic meters and $T$ is in $\mathrm{eV}$.

Thus, full recombination rate is the sum of these three terms

$R_{i}=R_{i}^{(p h)}+R_{i}^{(d)}+R_{i}^{(3 b)}$ 


\section{Radiation losses}

Radiation losses power is given by expression

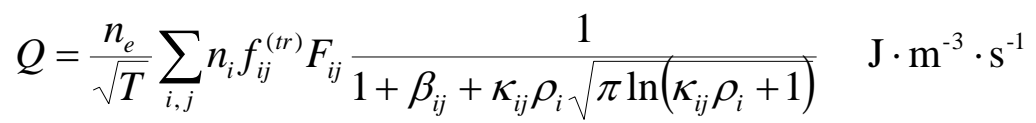

where (the unity in the argument of the logarithm in denominator is introduced to avoid floating exception in computer calculations)

$$
\begin{aligned}
& F_{i j}=6.98 \cdot 10^{-31} \cdot\left(1+\frac{3}{4} \frac{E_{i j}^{(t r)}}{T}\right) \cdot E i\left(\frac{E_{i j}^{(t r)}}{T}\right) \mathrm{J} \cdot \mathrm{m}^{-3} \cdot \mathrm{s}^{-1} \text {, if } \Delta n_{i j} \neq 0 \\
& F_{0 j}=C_{0}^{(r l 1)}\left(\frac{0.06}{1+E_{0 j}^{(r)} / T}\left(\sqrt{\left.\frac{E_{0 j}^{(r)}}{T}-2\right)}\right) \exp \left(-\frac{E_{0 j}^{(t r)}}{T}\right)+C_{0}^{(r l 2)} \cdot E i\left(\frac{E_{0 j}^{(r r)}}{T}\right)\right) \mathrm{J} \cdot \mathrm{m}^{-3} \cdot \mathrm{s}^{-1} \text {, if } \Delta n_{0 j}=0 \text { and } i=0 \\
& F_{i j}=C_{i}^{(r l 1)}\left(\exp \left(-\frac{E_{i j}^{(r)}}{T}\right)+C_{i}^{(r l 2)} \cdot E i\left(\frac{E_{i j}^{(r)}}{T}\right)\right) \mathrm{J} \cdot \mathrm{m}^{-3} \cdot \mathrm{s}^{-1} \text {, if } \Delta n_{i j}=0 \text { and } i>0 \\
& \left.\beta_{i j}=2.7 \cdot 10^{-19} \frac{n_{e}}{\left(E_{i j}^{(t r}\right)}\right)^{7 / 2} \sqrt{E_{i j}^{(t r)}}\left(1-\frac{E_{i j}^{(t r)}}{T} \exp \left(\begin{array}{c}
E_{i j}^{(t r)} \\
T
\end{array}\right) E i\left(\begin{array}{c}
E_{i j}^{(t r)} \\
T
\end{array}\right)\right) \\
& \kappa_{i j}=\pi n_{i}\left(\frac{\lambda_{i j}}{2 \pi}\right)^{2} \frac{1}{1+\Gamma / \gamma_{i j}}, \\
& \Gamma=7.30 \cdot 10^{-6} \sqrt{T(\mathrm{eV})} \text { for Argon only. }
\end{aligned}
$$$$
\gamma_{i j}=4.14 \cdot 10^{-15} \frac{v_{i j}\left(s^{-1}\right)}{E_{i j}^{(t)}(e V)} \text {, where } v_{i j} \text { is the reversed natural decay time (come from Verners tables [34]) }
$$

Thus

$$
\kappa_{i j}=\frac{1.23 \cdot 10^{-13} \cdot n_{i}}{\left(E_{i j}^{(t r)}(e V)\right)^{2}} \cdot \frac{1}{1+1.76 \cdot 10^{9} \frac{\sqrt{T} \cdot E_{i j}^{(t r)}(e V)}{v_{i j}\left(s^{-1}\right)}}
$$


Table 3

\begin{tabular}{|c|c|c|c|c|c|c|c|c|}
\hline$i$ & $j$ & $E^{(t r)}, \mathrm{eV}$ & $f^{(t r)}$ & $\Delta n$ & $C^{(\text {diel })}, 10^{-15} \mathrm{~m}^{3} / \mathrm{s}$ & $v, 10^{8} \mathrm{~s}^{-1}$ & $C_{i}^{(r l 1)} 10^{-30} \mathrm{Jm}^{3} \mathrm{~s}^{-1}$ & $C_{i}^{(r l 2)}$ \\
\hline $\begin{array}{l}0 \\
0\end{array}$ & $\begin{array}{l}1 \\
2 \\
\end{array}$ & $\begin{array}{l}11.9 \\
14.2\end{array}$ & $\begin{array}{l}0.310 \\
0.210\end{array}$ & $\begin{array}{l}1 \\
0\end{array}$ & $\begin{array}{l}0.00 \\
0.00\end{array}$ & $\begin{array}{l}4.94 \\
3.00\end{array}$ & $\begin{array}{l}2.50 \\
2.50\end{array}$ & $\begin{array}{l}0.276 \\
0.276\end{array}$ \\
\hline $\begin{array}{l}1 \\
1 \\
1 \\
1 \\
1 \\
1\end{array}$ & $\begin{array}{l}1 \\
2 \\
3 \\
4 \\
5 \\
6\end{array}$ & $\begin{array}{r}13.45 \\
17.17 \\
19.0 \\
21.5 \\
23.7 \\
25.7 \\
\end{array}$ & $\begin{array}{r}0.0180 \\
0.217 \\
0.241 \\
0.855 \\
0.669 \\
0.700 \\
\end{array}$ & $\begin{array}{l}0 \\
1 \\
1 \\
0 \\
1 \\
1\end{array}$ & $\begin{array}{r}0.0425 \\
0.261 \\
0.301 \\
0.00 \\
0.00 \\
0.00\end{array}$ & $\begin{array}{l}4.22 \\
27.6 \\
8.00 \\
6.40 \\
40.0 \\
32.0 \\
\end{array}$ & $\begin{array}{l}0.29 \\
0.29 \\
0.29 \\
0.29 \\
0.29 \\
0.29 \\
\end{array}$ & $\begin{array}{l}2.38 \\
2.38 \\
2.38 \\
2.38 \\
2.38 \\
2.38 \\
\end{array}$ \\
\hline $\begin{array}{l}2 \\
2 \\
2 \\
2 \\
2 \\
\end{array}$ & $\begin{array}{l}1 \\
2 \\
3 \\
4 \\
5 \\
\end{array}$ & $\begin{array}{r}14.14 \\
22.4 \\
25.74 \\
26.11 \\
26.56\end{array}$ & $\begin{array}{r}0.0385 \\
0.122 \\
0.897 \\
1.79 \\
0.355 \\
\end{array}$ & $\begin{array}{l}0 \\
1 \\
1 \\
0 \\
0\end{array}$ & $\begin{array}{r}0.275 \\
0.494 \\
3.83 \\
0.00 \\
0.00\end{array}$ & $\begin{array}{l}3.32 \\
79.2 \\
256 . \\
317 . \\
108 .\end{array}$ & $\begin{array}{l}1.00 \\
1.00 \\
1.00 \\
1.00 \\
1.00\end{array}$ & $\begin{array}{l}0.687 \\
0.687 \\
0.687 \\
0.687 \\
0.687\end{array}$ \\
\hline $\begin{array}{l}3 \\
3 \\
\end{array}$ & $\begin{array}{l}1 \\
2 \\
\end{array}$ & $\begin{array}{r}14.68 \\
27.5\end{array}$ & $\begin{array}{r}0.0774 \\
3.11 \\
\end{array}$ & $\begin{array}{l}0 \\
0\end{array}$ & $\begin{array}{r}1.09 \\
58.47 \\
\end{array}$ & $\begin{array}{l}2.40 \\
338 .\end{array}$ & $\begin{array}{l}1.33 \\
1.33\end{array}$ & $\begin{array}{l}0.519 \\
0.519\end{array}$ \\
\hline $\begin{array}{l}4 \\
4 \\
4 \\
4\end{array}$ & $\begin{array}{l}1 \\
2 \\
3 \\
4\end{array}$ & $\begin{array}{l}14.96 \\
17.46 \\
26.90 \\
27.75\end{array}$ & $\begin{array}{r}0.0043 \\
0.0607 \\
0.574 \\
1.80\end{array}$ & $\begin{array}{l}0 \\
0 \\
0 \\
0\end{array}$ & $\begin{array}{r}0.0010 \\
1.47 \\
17.0 \\
0.00\end{array}$ & $\begin{array}{l}2.48 \\
7.98 \\
180 . \\
250 .\end{array}$ & $\begin{array}{l}1.53 \\
1.53 \\
1.53 \\
1.53\end{array}$ & $\begin{array}{l}0.451 \\
0.451 \\
0.451 \\
0.451\end{array}$ \\
\hline $\begin{array}{l}5 \\
5 \\
5 \\
\end{array}$ & $\begin{array}{l}1 \\
2 \\
3\end{array}$ & $\begin{array}{l}16.29 \\
22.56 \\
27.02\end{array}$ & $\begin{array}{r}0.0661 \\
0.648 \\
1.10\end{array}$ & $\begin{array}{l}0 \\
0 \\
0\end{array}$ & $\begin{array}{l}2.20 \\
25.2 \\
46.5\end{array}$ & $\begin{array}{l}4.54 \\
143 . \\
170 .\end{array}$ & $\begin{array}{l}1.57 \\
1.57 \\
1.57 \\
\end{array}$ & $\begin{array}{l}0.441 \\
0.441 \\
0.441\end{array}$ \\
\hline $\begin{array}{l}6 \\
6 \\
\end{array}$ & $\begin{array}{l}1 \\
2\end{array}$ & $\begin{array}{l}21.21 \\
70.23\end{array}$ & $\begin{array}{l}1.240 \\
0.179\end{array}$ & $\begin{array}{l}0 \\
0\end{array}$ & $\begin{array}{l}61.8 \\
6.93 \\
\end{array}$ & $\begin{array}{l}80.4 \\
128 .\end{array}$ & $\begin{array}{l}1.64 \\
1.64\end{array}$ & $\begin{array}{l}0.421 \\
0.421\end{array}$ \\
\hline 7 & 1 & 17.66 & 0.573 & 0 & 32.9 & 25.7 & 1.55 & 0.445 \\
\hline $\begin{array}{l}8 \\
8 \\
8\end{array}$ & $\begin{array}{l}1 \\
2 \\
3\end{array}$ & $\begin{array}{l}255.2 \\
300.0 \\
355.1\end{array}$ & $\begin{array}{r}0.238 \\
2.01 \\
1.15\end{array}$ & $\begin{array}{l}1 \\
1 \\
1\end{array}$ & $\begin{array}{r}20.6 \\
177.0 \\
103.0\end{array}$ & $\begin{array}{l}2.23 \cdot 10^{3} \\
2.60 \cdot 10^{4} \\
2.50 \cdot 10^{3}\end{array}$ & $\begin{array}{l}0.00 \\
0.00 \\
0.00\end{array}$ & $\begin{array}{l}0.000 \\
0.000 \\
0.000\end{array}$ \\
\hline $\begin{array}{l}9 \\
9 \\
9 \\
9 \\
\end{array}$ & $\begin{array}{l}1 \\
2 \\
3 \\
4\end{array}$ & $\begin{array}{l}73.34 \\
323.7 \\
330.5 \\
340.0 \\
\end{array}$ & $\begin{array}{r}0.0663 \\
0.458 \\
1.454 \\
0.400\end{array}$ & $\begin{array}{l}0 \\
1 \\
1 \\
1\end{array}$ & $\begin{array}{r}10.7 \\
50.3 \\
160.0 \\
0.00 \\
\end{array}$ & $\begin{array}{r}475 \\
2.40 \cdot 10^{4} \\
2.50 \cdot 10^{4} \\
4.00 \cdot 10^{4}\end{array}$ & $\begin{array}{l}1.97 \\
1.97 \\
1.97 \\
1.97 \\
\end{array}$ & $\begin{array}{l}0.350 \\
0.350 \\
0.350 \\
0.350 \\
\end{array}$ \\
\hline $\begin{array}{l}10 \\
10\end{array}$ & $\begin{array}{l}1 \\
2\end{array}$ & $\begin{array}{l}65.77 \\
361.3 \\
\end{array}$ & $\begin{array}{r}0.115 \\
1.88 \\
\end{array}$ & $\begin{array}{l}0 \\
1\end{array}$ & $\begin{array}{l}20.70 \\
252.3 \\
\end{array}$ & $\begin{array}{r}215 \\
1.70 \cdot 10^{4}\end{array}$ & $\begin{array}{l}2.04 \\
2.04\end{array}$ & $\begin{array}{l}0.339 \\
0.339\end{array}$ \\
\hline $\begin{array}{l}11 \\
11\end{array}$ & $\begin{array}{l}1 \\
2 \\
\end{array}$ & $\begin{array}{l}56.24 \\
400.0\end{array}$ & $\begin{array}{r}0.145 \\
2.40 \\
\end{array}$ & $\begin{array}{l}0 \\
1\end{array}$ & $\begin{array}{r}28.0 \\
386.4 \\
\end{array}$ & $\begin{array}{r}66.2 \\
3.00 \cdot 10^{4}\end{array}$ & $\begin{array}{l}2.07 \\
2.07 \\
\end{array}$ & $\begin{array}{l}0.333 \\
0.333 \\
\end{array}$ \\
\hline $\begin{array}{l}12 \\
12 \\
12 \\
12 \\
\end{array}$ & $\begin{array}{l}1 \\
2 \\
3 \\
4 \\
\end{array}$ & $\begin{array}{l}50.74 \\
59.76 \\
76.26 \\
422.0 \\
\end{array}$ & $\begin{array}{r}0.0588 \\
0.0622 \\
0.0718 \\
1.58 \\
\end{array}$ & $\begin{array}{l}0 \\
0 \\
0 \\
1\end{array}$ & $\begin{array}{l}12.3 \\
14.5 \\
18.1 \\
0.00 \\
\end{array}$ & $\begin{array}{r}37.2 \\
96.0 \\
540 \\
4.21 \cdot 10^{4}\end{array}$ & $\begin{array}{l}2.19 \\
2.19 \\
2.19 \\
2.19\end{array}$ & $\begin{array}{l}0.315 \\
0.315 \\
0.315 \\
0.315 \\
\end{array}$ \\
\hline $\begin{array}{l}13 \\
13 \\
13\end{array}$ & $\begin{array}{l}1 \\
2 \\
3\end{array}$ & $\begin{array}{l}49.13 \\
66.83 \\
450.4\end{array}$ & $\begin{array}{r}0.0517 \\
0.130 \\
0.940\end{array}$ & $\begin{array}{l}0 \\
0 \\
1\end{array}$ & $\begin{array}{r}120.0 \\
34.8 \\
206.9\end{array}$ & $\begin{array}{r}32.4 \\
253 \\
2.50 \cdot 10^{4}\end{array}$ & $\begin{array}{l}2.13 \\
2.13 \\
2.13\end{array}$ & $\begin{array}{l}0.325 \\
0.325 \\
0.325\end{array}$ \\
\hline $\begin{array}{l}14 \\
14 \\
14\end{array}$ & $\begin{array}{l}1 \\
2 \\
3\end{array}$ & $\begin{array}{l}56.24 \\
503.2 \\
661.2\end{array}$ & $\begin{array}{l}0.205 \\
0.636 \\
0.166\end{array}$ & $\begin{array}{l}0 \\
1 \\
2\end{array}$ & $\begin{array}{r}56.4 \\
161.8 \\
43.02\end{array}$ & $\begin{array}{r}93.2 \\
2.31 \cdot 10^{4} \\
1.04 \cdot 10^{4}\end{array}$ & $\begin{array}{l}2.15 \\
2.15 \\
2.15\end{array}$ & $\begin{array}{l}0.310 \\
0.310 \\
0.310\end{array}$ \\
\hline 15 & 1 & 34.06 & 0.0842 & 0 & 201.6 & 14.10 & 2.89 & 0.231 \\
\hline $\begin{array}{l}16 \\
16 \\
16\end{array}$ & $\begin{array}{l}1 \\
2 \\
3\end{array}$ & $\begin{array}{l}3147 \\
3688 \\
3844\end{array}$ & $\begin{array}{r}0.775 \\
0.155 \\
0.0573\end{array}$ & $\begin{array}{l}1 \\
1 \\
1\end{array}$ & $\begin{array}{r}212.8 \\
40.6 \\
14.8\end{array}$ & $\begin{array}{l}1.11 \cdot 10^{6} \\
3.04 \cdot 10^{5} \\
1.24 \cdot 10^{5}\end{array}$ & $\begin{array}{l}0.00 \\
0.00 \\
0.00\end{array}$ & $\begin{array}{l}0.000 \\
0.000 \\
0.000\end{array}$ \\
\hline $\begin{array}{l}17 \\
17 \\
17\end{array}$ & $\begin{array}{l}1 \\
2 \\
3\end{array}$ & $\begin{array}{l}3332 \\
3946 \\
4157\end{array}$ & $\begin{array}{l}0.416 \\
0.079 \\
0.029\end{array}$ & $\begin{array}{l}1 \\
1 \\
1\end{array}$ & $\begin{array}{r}128.1 \\
23.1 \\
8.356\end{array}$ & $\begin{array}{l}6.64 \cdot 10^{5} \\
1.77 \cdot 10^{5} \\
7.22 \cdot 10^{4}\end{array}$ & $\begin{array}{l}0.00 \\
0.00 \\
0.00\end{array}$ & $\begin{array}{l}0.000 \\
0.000 \\
0.000\end{array}$ \\
\hline
\end{tabular}

